\title{
SOBOLEV ORTHOGONAL POLYNOMIALS ON A SIMPLEX
}

\author{
RABİA AKTAŞ AND YUAN XU
}

\begin{abstract}
The Jacobi polynomials on the simplex are orthogonal polynomials with respect to the weight function $W_{\boldsymbol{\gamma}}(x)=x_{1}^{\gamma_{1}} \cdots x_{d}^{\gamma_{d}}(1-|x|)^{\gamma_{d+1}}$ when all $\gamma_{i}>-1$ and they are eigenfunctions of a second order partial differential operator $L_{\boldsymbol{\gamma}}$. The singular cases that some, or all, $\gamma_{1}, \ldots, \gamma_{d+1}$ are -1 are studied in this paper. Firstly a complete basis of polynomials that are eigenfunctions of $L_{\gamma}$ in each singular case is found. Secondly, these polynomials are shown to be orthogonal with respect to an inner product which is explicitly determined. This inner product involves derivatives of the functions, hence the name Sobolev orthogonal polynomials.
\end{abstract}

\section{INTRODUCTION}

The purpose of this paper is to study the limiting case of classical orthogonal polynomials on the simplex when the weight function becomes singular. Let $T^{d}$ be the $d$-dimensional simplex defined by

$$
T^{d}:=\left\{x \in \mathbb{R}^{d}: x_{1} \geq 0, \ldots, x_{d} \geq 0,1-|x| \geq 0\right\},
$$

where $|x|:=x_{1}+\cdots+x_{d}$. The classical weight function on $T^{d}$ is defined by

$$
W_{\gamma}(x):=x_{1}^{\gamma_{1}} \cdots x_{d}^{\gamma_{d}}(1-|x|)^{\gamma_{d+1}}, \quad x \in T^{d},
$$

where $\gamma_{i}$ are real numbers, usually assumed to satisfy $\gamma_{i}>-1$ to ensure the integrability of $W_{\gamma}$ on $T^{d}$. Let $c_{\gamma}=1 / \int_{T^{d}} W_{\gamma}(x) d x$ denote the normalization constant.

Let $\Pi^{d}=\mathbb{R}[x]$ be the ring of polynomials in $d$-variables and let $\Pi_{n}^{d}$ denote the subspace of polynomials of total degree at most $n$. When all $\gamma_{i}>-1$, the bilinear form

$$
\langle f, g\rangle_{\gamma}:=c_{\gamma} \int_{T^{d}} f(x) g(x) W_{\gamma}(x) d x
$$

defines an inner product on the space $\Pi^{d}$ of polynomials of $d$-variables. The orthogonal polynomials with respect to this inner product have been studied extensively (cf. [7). In particular, let $\mathcal{V}_{n}^{d}\left(W_{\gamma}\right)$ denote the space of orthogonal polynomials of

Date: March 23, 2022.

2000 Mathematics Subject Classification. 33C50, 33E30,42C05.

Key words and phrases. Sobolev orthogonal polynomials, simplex, eigenfunctions, partial differential operators.

The work of the second author was supported in part by NSF Grant DMS-1106113. 
degree $n$ with respect to $\langle\cdot, \cdot\rangle_{\gamma}$. Then, for all $P \in \mathcal{V}_{n}^{d}\left(W_{\gamma}\right)$,

$$
\begin{aligned}
L_{\gamma} P:= & \sum_{i=1}^{d} x_{i}\left(1-x_{i}\right) \frac{\partial^{2} P}{\partial x_{i}^{2}}-2 \sum_{1 \leq i<j \leq d} x_{i} x_{j} \frac{\partial^{2} P}{\partial x_{i} \partial x_{j}} \\
& +\sum_{i=1}^{d}\left(\gamma_{i}+1-(|\gamma|+d+1) x_{i}\right) \frac{\partial P}{\partial x_{i}}=-n(n+|\gamma|+d) P
\end{aligned}
$$

where $|\gamma|:=\gamma_{1}+\cdots+\gamma_{d+1}$. In other words, orthogonal polynomials of degree $n$ are eigenfunctions of the differential operator $L_{\boldsymbol{\gamma}}$.

If some or all $\gamma_{i}$ equal to -1 , the weight function becomes singular and orthogonal polynomials with respect to $W_{\gamma}$ are no longer well defined. In fact, the Jacobi polynomials on the simplex defined via the Rodrigue formula can be extended to the case of $\gamma_{i} \leq-1$. In the case of all $\gamma_{i}=-1$, they have been used to study certain approximation processes in [14, 16, but this family of polynomials does not provide a complete basis. On the other hand, the equation (1.2) still makes sense if some, or all, $\gamma_{i}=-1$. Thus, it is natural to ask two questions: firstly if (1.2) still has a complete basis of polynomial solutions, and secondly, when the answer to the first question is affirmative, if these polynomial solutions are orthogonal with respect to an inner product.

The main results of this paper answer both questions affirmatively. More precisely, for each singular case of some or all $\gamma_{i}=-1$, we have identified a complete basis of polynomials that are eigenfunctions of $L_{\gamma}$, which are given explicitly, and we have found an inner product explicitly, with respect to which these polynomials are orthogonal. The inner product turns out to involve derivatives of the functions, so that the orthogonality is not established in the usual $L^{2}\left(W_{\gamma}\right)$ sense, but in a Sobolev space. Such orthogonal polynomials are named Sobolev orthogonal polynomials.

The problems are motivated by a recent study [13, 18 of analogue problems on the unit ball, for which the classical weight function takes the form $W_{\mu}(x)=$ $\left(1-\|x\|^{2}\right)^{\mu}$. The orthogonal polynomials with respect to $W_{\mu}$ on the ball are eigenfunctions of a second order differential operator $L_{\mu}$. In [18, a family of orthogonal polynomials with respect to an inner product that involves the first partial derivatives on the unit ball was studied, which turned out to be the eigenfunctions of $L_{-1}$ as shown in [13.

The Sobolev orthogonal polynomials have been studied extensively when $d=1$ (cf. 9]). The simplex $T^{d}$ becomes, when $d=1$, the interval $[0,1]$ and $W_{\gamma}$ is the Jacobi weight function $x^{\alpha}(1-x)^{\beta}$, where we have written $\alpha=\gamma_{1}$ and $\beta=$ $\gamma_{2}$. In this case, Sobolev orthogonal polynomials have been studied by several authors (11, 2, 4, 11). More precisely, the Sobolev orthogonality of the Jacobi polynomials $\left\{P_{n}^{(-N, \beta)}\right\}_{n \geq 0}$ was studied in [3], and the case $\left\{P_{n}^{(-N,-N)}\right\}_{n \geq 0}$ was studied in [4, in both cases $N$ is a positive integer. The particular case of the Jacobi polynomials $\left\{P_{n}^{(-1,-1)}\right\}_{n \geq 0}$ had been previously given in [11]. In the case of $d=2$, the simplex becomes a triangle and the weight function is usually denoted by $W_{\alpha, \beta, \gamma}$. An observation in [6] shows that the monic basis for $W_{\alpha, \beta, \gamma}$ is still a basis when $\gamma=-1$ and $\alpha, \beta>-1$, and they are orthogonal with respect to an inner product that involves the first order derivatives. In contrast to one variable, there are only a handful papers on Sobolev orthogonal polynomials of several variables 
6, 10, 12, 13, 17, 18, see also [8. It should be mentioned that the studies in [17, 18, are motivated by problems in numerical solution of Poisson equations [5] and in optics. Given this background, it is somewhat surprising that there have been so few studies of the Sobolev orthogonal polynomials of several variables. It is our hope that the results in this paper will help to kindle more interests on this topic.

The paper is organized as follows. In the next section we recall results on Jacobi polynomials and classical orthogonal polynomials on the simplex, where we will also present several new properties on the simplex, including how orthogonal bases on the faces of $T^{d}$ arise from some of the orthogonal polynomials on $T^{d}$, which are of independent interest. The main results are stated in the third section, where we will state the results for $d=2$ first to illustrate the results and illuminate how the results are obtained, as the results in $T^{d}$ require unavoidably heavy notations. Finally, the proofs of the main results, including several lemmas, are given in the fourth section.

\section{ORThOGONAL POLYNOMIALS ON THE SIMPLEX}

This section contains background results on orthogonal polynomials that are necessary for latter sections. After a brief subsection on the classical Jacobi polynomials, orthogonal polynomials on the triangle are described in the second subsection and those on the simplex are developed in full generality in the third subsection, and orthogonal polynomials on the faces of the simplex are discussed in the fourth subsection.

2.1. Jacobi polynomials. The Jacobi weight function $v_{\alpha, \beta}$ is defined by

$$
v_{\alpha, \beta}(x):=(1-x)^{\alpha}(1+x)^{\beta}, \quad \alpha, \beta>-1 .
$$

The Jacobi polynomials are given explicitly by the Rodrigue formula

$$
P_{n}^{(\alpha, \beta)}(x)=\frac{(-1)^{n}}{2^{n} n !}(1-x)^{-\alpha}(1+x)^{-\beta} \frac{d^{n}}{d x^{n}}\left[(1-x)^{n+\alpha}(1+x)^{n+\beta}\right]
$$

and they satisfy the orthogonality condition

$$
c_{\alpha, \beta} \int_{-1}^{1} P_{n}^{(\alpha, \beta)}(x) P_{m}^{(\alpha, \beta)}(x) v_{\alpha, \beta}(x) d x=h_{n}^{(\alpha, \beta)} \delta_{n, m},
$$

where

$$
c_{\alpha, \beta}:=\frac{\Gamma(\alpha+\beta+2)}{2^{\alpha+\beta+1} \Gamma(\alpha+1) \Gamma(\beta+1)}, \quad h_{n}^{(\alpha, \beta)}:=\frac{(\alpha+1)_{n}(\beta+1)_{n}(\alpha+\beta+n+1)}{n !(\alpha+\beta+2)_{n}(\alpha+\beta+2 n+1)}
$$

and $\delta_{n, m}$ is the Kronecker delta. The Jacobi polynomial $P_{n}^{(\alpha, \beta)}$ also satisfies the second order differential equation

$$
\left(1-x^{2}\right) y^{\prime \prime}+[\beta-\alpha-(\alpha+\beta+2) x] y^{\prime}=-n(\alpha+\beta+n+1) y,
$$

in other words, it is the eigenfunction of the differential operator in the left hand side with the eigenvalue $-n(\alpha+\beta+n+1)$.

If one of $\alpha$ and $\beta$ is a negative integer, then the weight function $v_{\alpha, \beta}$ is no longer integrable on $[-1,1]$. Assume $\alpha=-l, l \in \mathbb{N}$, then we have [15, (4.22.2)]

$$
\left(\begin{array}{c}
n \\
l
\end{array}\right) P_{n}^{(-l, \beta)}(x)=\left(\begin{array}{c}
n+\beta \\
l
\end{array}\right)\left(\frac{x-1}{2}\right)^{l} P_{n-l}^{(l, \beta)}(x),
$$


which is well defined for $n \geq l$. In the case of $\alpha=-1$, defining $P_{0}^{(-1, \beta)}(x)=1$, then $\left\{P_{n}^{(-1, \beta)}\right\}_{n \geq 0}$ is well defined and they still satisfy the differential equation (2.3) for all $n=0,1, \ldots$. They are, however, no longer orthogonal polynomials in the sense of (2.2) but, as it turns out, orthogonal polynomials with respect to the following Sobolev type inner product [3, 11,

$$
\langle f, g\rangle:=\lambda f(1) g(1)+\int_{-1}^{1}(x+1)^{\beta+1} f^{\prime}(x) g^{\prime}(x) d x, \quad \lambda>0, \quad \beta>-1 .
$$

If both $\alpha=\beta=-1$, then the Jacobi polynomials in (2.1) are well defined for $n \geq 2$. Furthermore, any linear polynomial will be a solution of the equation (2.3) when $n=0$ and 1 . In fact, the polynomials $P_{0}^{(-1,-1)}(x)=1, P_{1}^{(-1,-1)}(x)=x+\mu$ and

$$
P_{n}^{(-1,-1)}(x)=\frac{1}{4}(x-1)(x+1) P_{n-2}^{(1,1)}(x), \quad n \geq 2,
$$

satisfy the equation (2.3) for all $n=0,1,2, \ldots$. Furthermore, these polynomials are orthogonal with respect to the Sobolev type inner product

$$
\langle f, g\rangle:=\lambda_{1} f(1) g(1)+\lambda_{2} f(-1) g(-1)+\int_{-1}^{1} f^{\prime}(x) g^{\prime}(x) d x
$$

where $\lambda_{1}$ and $\lambda_{2}$ are nonnegative numbers, not both zero, and the constant in $P_{1}^{(-1,-1)}(x)$ is given by $\mu=\frac{\lambda_{2}-\lambda_{1}}{\lambda_{1}+\lambda_{2}}$.

Since the simplex $T^{d}$ becomes $[0,1]$ when $d=1$, it is more convenient to consider the Jacobi polynomials on the interval $[0,1]$, which we denote by $J_{n}^{(\alpha, \beta)}$, and normalized as

$$
J_{n}^{(\alpha, \beta)}(x):=(1-x)^{-\alpha} x^{-\beta} \frac{d^{n}}{d x^{n}}\left[(1-x)^{n+\alpha} x^{n+\beta}\right] .
$$

Up to a constant multiple, $J_{n}^{(\alpha, \beta)}(x)=c P_{n}^{(\alpha, \beta)}(2 x-1)$. These polynomials are orthogonal on $[0,1]$ with respect to the weight function

$$
w_{a, \beta}(x):=(1-x)^{\alpha} x^{\beta}, \quad \alpha, \beta>-1
$$

and they satisfy a second order differential equation

$$
x(1-x) y^{\prime \prime}+[\beta+1-(\alpha+\beta+2) x] y^{\prime}=-n(n+\alpha+\beta+1) y
$$

2.2. Orthogonal polynomials on the triangle. On the triangle $T^{2}:=\{(x, y) \in$ $\left.\mathbb{R}^{2}: x, y \geq 0,1-x-y \geq 0\right\}$, the Jacobi polynomials are orthogonal with respect to the weight function

$$
W_{\alpha, \beta, \gamma}(x, y):=x^{\alpha} y^{\beta}(1-x-y)^{\gamma}, \quad \alpha, \beta, \gamma>-1 .
$$

More precisely, we define the inner product

$$
\langle f, g\rangle_{\alpha, \beta, \gamma}:=c_{\alpha, \beta, \gamma} \int_{T^{2}} f(x, y) g(x, y) W_{\alpha, \beta, \gamma}(x, y) d x d y,
$$

where $c_{\alpha, \beta, \gamma}$ is the normalization constant of $W_{\alpha, \beta, \gamma}$ given by

$$
c_{\alpha, \beta, \gamma}:=1 / \int_{T^{2}} W_{\alpha, \beta, \gamma}(x, y) d x d y=\frac{\Gamma(\alpha+\beta+\gamma+3)}{\Gamma(\alpha+1) \Gamma(\beta+1) \Gamma(\gamma+1)} .
$$

Let $\mathcal{V}_{n}^{2}\left(W_{\alpha, \beta, \gamma}\right)$ denote the space of orthogonal polynomials of degree $n$ with respect to this inner product. Then $P \in \mathcal{V}_{n}^{2}\left(W_{\alpha, \beta, \gamma}\right)$ if $\langle P, q\rangle_{\alpha, \beta, \gamma}=0$ for all $q \in \Pi_{n-1}^{2}$ and 
$\operatorname{dim} \mathcal{V}_{n}^{2}\left(W_{\alpha, \beta, \gamma}\right)=(n+1)$. Among many bases for $\mathcal{V}_{n}^{2}\left(W_{\alpha, \beta, \gamma}\right)$, one is given by the Rodrigue formula: For $0 \leq k \leq n$,

$$
P_{k, n}^{(\alpha, \beta, \gamma)}(x, y):=\left[W_{\alpha, \beta, \gamma}(x, y)\right]^{-1} \frac{\partial^{n}}{\partial x^{k} \partial y^{n-k}}\left[x^{\alpha+k} y^{\beta+n-k}(1-x-y)^{\gamma+n}\right] .
$$

It should be noted that the elements of this basis are not mutually orthogonal to each other. The triangle $T^{2}$ is symmetric under the permutation of $(x, y, 1-x-$ $y$ ). Parametrizing the triangle differently leads to two more orthogonal bases of $\mathcal{V}_{n}^{2}\left(W_{\alpha, \beta, \gamma}\right)$. Indeed, if we define

$$
Q_{k}^{n}(x, y):=P_{k, n}^{(\beta, \alpha, \gamma)}(y, x) \quad \text { and } \quad R_{k}^{n}(x, y):=P_{k, n}^{(\gamma, \beta, \alpha)}(1-x-y, y),
$$

then both $\left\{R_{k}^{n}: 0 \leq k \leq n\right\}$ and $\left\{Q_{k}^{n}: 0 \leq k \leq n\right\}$ are bases of $\mathcal{V}_{n}^{2}\left(W_{\alpha, \beta, \gamma}\right)$. Upon changing variables from (2.5), these polynomials are given explicitly by

$$
Q_{k}^{n}(x, y)=\left[W_{\alpha, \beta, \gamma}(x, y)\right]^{-1} \frac{\partial^{n}}{\partial x^{n-k} \partial y^{k}}\left[x^{\alpha+n-k} y^{\beta+k}(1-x-y)^{\gamma+n}\right]
$$

and

$$
R_{k}^{n}(x, y)=(-1)^{k}\left[W_{\alpha, \beta, \gamma}(x, y)\right]^{-1} \frac{\partial^{n}}{\partial x^{k}(\partial y-\partial x)^{n-k}}\left[y^{\beta+n-k}(1-x-y)^{\gamma+k} x^{\alpha+n}\right] .
$$

For the study of the Sobolev orthogonal polynomials, it is necessary to understand the restriction of the basis element on the boundary of the triangle. For later use, we state the following proposition, which is the special case of Lemma 2.10.

Proposition 2.1. For $n=0,1, \ldots$,

1. The restriction of $P_{0, n}^{(\alpha, \beta, \gamma)} \in \mathcal{V}_{n}^{2}\left(W_{\alpha, \beta, \gamma}\right)$ on the line $x=0$ is $J_{n}^{(\gamma, \beta)}(y)$.

2. The restriction of $P_{0, n}^{(\beta, \alpha, \gamma)} \in \mathcal{V}_{n}^{2}\left(W_{\alpha, \beta, \gamma}\right)$ on the line $y=0$ is $J_{n}^{(\gamma, \alpha)}(x)$.

3. The restriction of $P_{0, n}^{(\gamma, \beta, \alpha)} \in \mathcal{V}_{n}^{2}\left(W_{\alpha, \beta, \gamma}\right)$ on the line $x+y=1$ is $J_{n}^{(\alpha, \beta)}(y)$.

There is another basis $\left\{V_{k, n}^{(\alpha, \beta, \gamma)}: 0 \leq k \leq n\right\}$ of $\mathcal{V}_{n}^{2}\left(W_{\alpha, \beta, \gamma}\right)$ so that $P_{k, n}^{(\alpha, \beta, \gamma)}$ and $V_{k, n}^{(\alpha, \beta, \gamma)}$ are biorthogonal. This basis will be the special case of $d=2$ of the basis (2.11) in the following section.

2.3. Orthogonal polynomials on the simplex. To simplify the notation, we set, for $x \in T^{d},|x|:=x_{1}+\cdots+x_{d}$ and $x_{d+1}:=1-|x|$. Furthermore, for $\gamma_{i}>-1$, $1 \leq i \leq d+1$, we set

$$
\gamma:=\left(\gamma_{1}, \ldots, \gamma_{d}, \gamma_{d+1}\right)=\left(\gamma, \gamma_{d+1}\right) \quad \text { with } \quad \gamma:=\left(\gamma_{1}, \ldots, \gamma_{d}\right) .
$$

Then the weight function $W_{\gamma}$ in (1.1) can be written as

$$
W_{\gamma}(x)=x^{\gamma}(1-|x|)^{\gamma_{d+1}} \text {. }
$$

If all $\gamma_{i}>-1$, then $W_{\gamma}$ is integrable and we can consider orthogonal polynomials in the space $L^{2}\left(W_{\gamma}, T^{d}\right)$ with respect to the inner product

$$
\langle f, g\rangle_{\gamma}:=c_{\gamma} \int_{T^{d}} f(x) g(x) W_{\gamma}(x) d x,
$$

where $c_{\gamma}$ is the normalization constant of $W_{\gamma}$ given by

$$
c_{\gamma}:=1 / \int_{T^{d}} W_{\gamma}(x) d x=\frac{\Gamma(|\gamma|+d+1)}{\prod_{i=1}^{d+1} \Gamma\left(\gamma_{i}+1\right)} .
$$


Let $\mathcal{V}_{n}^{d}\left(W_{\gamma}\right)$ denote the space of orthogonal polynomials of degree $n$ with respect to this inner product. As in the case of two variables, there are several distinguished bases of $\mathcal{V}_{n}^{d}\left(W_{\gamma}\right)$. First we state the basis defined via the Rodrigue formula.

Lemma 2.2. For $\mathbf{n} \in \mathbb{N}_{0}^{d}, \gamma \in \mathbb{R}^{d+1}$ and $x \in \mathbb{R}^{d}$, define

$$
P_{\mathbf{n}}^{\gamma}(x):=x^{-\gamma}(1-|x|)^{-\gamma_{d+1}} \frac{\partial^{|\mathbf{n}|}}{\partial x^{\mathbf{n}}}\left[x^{\gamma+\mathbf{n}}(1-|x|)^{\gamma_{d+1}+|\mathbf{n}|}\right],
$$

where $\frac{\partial^{|\mathbf{n}|}}{\partial x^{\mathbf{n}}}=\frac{\partial^{|\mathbf{n}|}}{\partial x_{1}^{n_{1} \ldots \partial x_{d}^{n} d}}$. If $\gamma_{i}>-1,1 \leq i \leq d+1$, then $P_{\mathbf{n}}^{\gamma}$ are orthogonal polynomials with respect to $W_{\boldsymbol{\gamma}}$ and $\left\{P_{\mathbf{n}}^{\gamma}:|\mathbf{n}|=n\right\}$ is a basis of $\mathcal{V}_{n}^{d}\left(W_{\gamma}\right)$.

This is a classical result, see, for example, [7, p. 49]. As a basis of $\mathcal{V}_{n}^{d}\left(W_{\gamma}\right)$, these polynomials are eigenfunctions of the differential operator $L_{\gamma}$ in (1.2); that is,

$$
L_{\gamma} P_{\mathbf{n}}^{\gamma}=-n(n+|\gamma|+d) P_{\mathbf{n}}^{\gamma}, \quad|\mathbf{n}|=n .
$$

In particular, the differential equation has a complete set of solutions consisting of $\left(\begin{array}{c}n+d-1 \\ n\end{array}\right)$ linearly independent polynomials of degree $n$.

Remark 2.1. Analytic continuation shows that (2.10) still holds if some or all $\gamma_{i}$ are $\leq-1$, in which case, however, $\left\{P_{\mathbf{n}}^{\gamma}:|\mathbf{n}|=n\right\}$ no longer contains a complete set of solutions.

If some components of $\gamma$ are negative integers, the polynomial $P_{\mathbf{n}}^{\gamma}$ still satisfies some orthogonality with respect to a different weight function. More precisely, we have the following lemma.

Lemma 2.3. Let $k$ be an integer, $1 \leq k \leq d$, and $\gamma \in \mathbb{R}^{d-k+1}$ with $\gamma_{i}>-1$ for all $i$. Let $\boldsymbol{\gamma}=(\gamma,-\mathbf{m})$, where $\mathbf{m} \in \mathbb{N}^{k}$. Then $P_{\mathbf{n}}^{\boldsymbol{\gamma}}$ is orthogonal to polynomials of degree at most $|\mathbf{n}|-|\mathbf{m}|-1$ with respect to $W_{\gamma, \mathbf{0}_{k}}$; that is,

$$
\int_{T^{d}} P_{\mathbf{n}}^{(\gamma,-\mathbf{m})}(x) Q(x) W_{\gamma, \mathbf{0}_{k}}(x) d x=0, \quad \forall Q \in \Pi_{|\mathbf{n}|-|\mathbf{m}|-1}^{d} .
$$

Proof. Let us denote temporarily $x=\left(\mathbf{x}_{1}, \mathbf{x}_{2}\right)$ with $\mathbf{x}_{1} \in \mathbb{R}^{d-k+1}$ and $\mathbf{x}_{2} \in \mathbb{R}^{k-1}$ and, similarly, $\mathbf{n}=\left(\mathbf{n}_{1}, \mathbf{n}_{2}\right)$. Furthermore, let $\mathbf{m}=\left(\mathbf{m}^{\prime}, m_{0}\right)$ with $\mathbf{m}^{\prime} \in \mathbb{N}^{k-1}$. Then, by the Rodrigue formula (2.9),

$$
\begin{aligned}
J:=\int_{T^{d}} P_{\mathbf{n}}^{(\gamma,-\mathbf{m})}(x) Q(x) W_{\gamma, \mathbf{0}_{k}}(x) d x \\
\quad=\int_{T^{d}} \mathbf{x}_{2}^{\mathbf{m}^{\prime}}(1-|x|)^{m_{0}} \frac{\partial^{|\mathbf{n}|}}{\partial x^{\mathbf{n}}}\left\{\mathbf{x}_{1}^{\mathbf{n}_{1}+\gamma} \mathbf{x}_{2}^{\mathbf{n}_{2}-\mathbf{m}^{\prime}}(1-|x|)^{|\mathbf{n}|-m_{0}}\right\} Q(x) d x .
\end{aligned}
$$

We perform integration by parts on the last integral. Since $\gamma_{i}>-1$, the integration by parts over the first $d-k+1$ variables carries through without problem. For integration by parts in $x_{d-k+2}$, the first component of $\mathbf{x}_{2}$, the part that is being integrated out, is zero since, if $j \leq m_{1}$, then $\frac{\partial^{j-1}}{\partial x_{d-k+2}^{j-1}}\left[\mathbf{x}_{2}^{\mathbf{m}^{\prime}}(1-|x|)^{m_{0}} Q(x)\right]$ vanishes for $x_{d-k+2}=0$ and $|x|=1$, whereas if $j>m_{1}$, then for $\ell=n_{d-k+2}-j$, $\frac{\partial^{\ell}}{\partial x_{d-k+2}^{\ell}}\left\{\mathbf{x}_{2}^{\mathbf{n}_{2}-\mathbf{m}^{\prime}}(1-|x|)^{|\mathbf{n}|-m_{0}}\right\}$ vanishes on the boundary. Consequently, we conclude that

$$
J=(-1)^{|\mathbf{n}|} \int_{T^{d}} \mathbf{x}_{1}^{\mathbf{n}_{1}+\gamma} \mathbf{x}_{2}^{\mathbf{n}_{2}-\mathbf{m}^{\prime}}(1-|x|)^{|\mathbf{n}|-m_{0}} \frac{\partial^{|\mathbf{n}|}}{\partial x^{\mathbf{n}}}\left[\mathbf{x}_{2}^{\mathbf{m}^{\prime}}(1-|x|)^{m_{0}} Q(x)\right] d x=0
$$

since the term insider the bracket is a polynomial of degree $|\mathbf{m}|+\operatorname{deg} Q<n$. 
It should be noted that, instead of $\gamma=(\gamma,-\mathbf{m})$, we can assume that $\gamma_{i}$ is a negative integer for $i$ in a subset of $\{1,2, \ldots, d+1\}$ in the above lemma. The result will be similar and the precise formulation should be clear from the above proof.

The simplex $T^{d}$ is symmetric under the permutation of $\left(x_{1}, \ldots, x_{d}, x_{d+1}\right)$. This symmetry carries over to the Rodrigue basis. Let

$$
\mathbb{Z}_{d}:=\{1,2, \ldots, d\}
$$

For a subset $S$ of $\mathbb{Z}_{d}$ we denote by $|S|$ the cardinality of $S$ and by $S^{c}$ the complement of $S$, that is, $S^{c}:=\mathbb{Z}_{d} \backslash S$.

Let $\mathcal{G}_{k}$ be the permutation group of $k$ elements; its action on a function $g$ : $\mathbb{R}^{k} \mapsto \mathbb{R}$ is denoted by $g(x \sigma), \sigma \in \mathcal{G}_{k}$. Just as in the case of $d=2$, see (2.6), we can permute variables and parameters of $P_{\mathbf{n}}^{\boldsymbol{\gamma}}$ to obtain different bases of $\mathcal{V}_{n}^{d}\left(W_{\boldsymbol{\gamma}}\right)$.

Definition 2.4. Let $S=\left\{i_{1}, \ldots, i_{d}\right\}$ be a subset of $\mathbb{Z}_{d+1}$ and $S^{c}=\left\{i_{d+1}\right\}$. Let $\gamma_{S}:=\left(\gamma_{S}, \gamma_{i_{d+1}}\right)$, where $\gamma_{S}:=\left(\gamma_{i_{1}}, \ldots, \gamma_{i_{d}}\right)$, and $x_{S}=\left(x_{i_{1}}, \ldots, x_{i_{d}}\right)$.

1. If $d+1 \notin S$, then $S^{c}=\{d+1\}, x_{S}=\left(x_{1}, \ldots, x_{d}\right) \sigma$ and $\gamma_{S}=\left(\gamma_{1}, \ldots, \gamma_{d}\right) \sigma$ for some $\sigma \in \mathcal{G}_{d}$, and $\gamma_{S}=\left(\gamma_{S}, \gamma_{d+1}\right)$. We denote

$$
P_{\mathbf{n}}^{\boldsymbol{\gamma}_{S}}\left(x_{S}\right)=x_{S}^{-\gamma_{S}}\left(1-\left|x_{S}\right|\right)^{-\gamma_{d+1}} \frac{\partial^{|\mathbf{n}|}}{\partial x_{S}^{\mathbf{n}}}\left\{x_{S}^{\gamma_{S}+\mathbf{n}}\left(1-\left|x_{S}\right|\right)^{\gamma_{d+1}+|\mathbf{n}|}\right\} .
$$

2. If $d+1 \in S$, then $S=\left\{d+1, i_{2}, \ldots, i_{d}\right\} \sigma$ for some $\sigma \in \mathcal{G}_{d}$ and $S^{c}=\left\{i_{d+1}\right\}$, where $i_{j} \in \mathbb{Z}_{d}$. Let $x_{S}=\left(x_{d+1}, x_{i_{2}}, \ldots, x_{i_{d}}\right) \sigma$ and $\gamma_{S}=\left(\gamma_{d+1}, \gamma_{i_{2}}, \ldots, \gamma_{i_{d}}\right) \sigma$. We define then

$$
P_{\mathbf{n}}^{\gamma_{S}}\left(x_{S}\right)=x_{S}^{-\gamma_{S}} x_{i_{d+1}}^{-\gamma_{i+1}} \frac{\partial^{|\mathbf{n}|}}{\partial \mathbf{x}_{S, \sigma}^{\mathbf{n}}}\left\{x_{S}^{\gamma_{S}+\mathbf{n}} x_{i_{d+1}}^{\gamma_{i d+1}+|\mathbf{n}|}\right\}
$$

where $\partial \mathbf{x}_{S, \sigma}=\left(-\partial_{i_{d+1}}, \partial_{i_{2}, i_{d+1}}, \ldots, \partial_{i_{d}, i_{d+1}}\right) \sigma$ with $\partial_{i}:=\partial x_{i}$ and $\partial_{i, j}:=\partial_{i}-\partial_{j}$.

In the case of $d+1 \in S$, the notation $P_{\mathbf{n}}^{\gamma_{S}}\left(x_{S}\right)$ agrees with that of (2.9), and it is a simple permutation of $P_{\mathbf{n}}^{\gamma}(x)$. For $d=2$, see (2.6).

Lemma 2.5. Let $S$ and $P_{\mathbf{n}}^{\boldsymbol{\gamma}_{S}}\left(x_{S}\right)$ be defined in the Definition 2.4. Then $\left\{P_{\mathbf{n}}^{\boldsymbol{\gamma}_{S}}\left(x_{S}\right)\right.$ : $|\mathbf{n}|=n\}$ is a basis of $\mathcal{V}_{n}^{d}\left(W_{\boldsymbol{\gamma}}\right)$. In particular, $P_{\mathbf{n}}^{\boldsymbol{\gamma}_{S}}\left(x_{S}\right)$ are eigenfunctions of the differential operator $L_{\boldsymbol{\gamma}}$ given by (1.2).

Proof. In the first case, $d+1 \notin S$, changing variable $x \mapsto x \sigma$ in the integral of $\left\langle P_{\mathbf{n}}, g\right\rangle_{\boldsymbol{\gamma}}$ shows immediately that $P_{\mathbf{n}}^{\boldsymbol{\gamma}_{S}}\left(x_{S}\right) \in \mathcal{V}_{n}^{d}\left(W_{\boldsymbol{\gamma}}\right)$

The second case, $d+1 \in S$, requires a bit more work. Up to a permutation in $\mathcal{G}_{d}$, we can assume that $S=(d+1,1, \ldots, d-1)$. Then $S^{c}=\{d\}, x_{S}=$ $\left(x_{d+1}, x_{1}, \ldots, x_{d-1}\right)$ and $\gamma_{S}=\left(\gamma_{d+1}, \gamma_{1}, \ldots, \gamma_{d-1}\right)$. Making a change of variable

$$
u_{1}=x_{d+1}=1-|x|, \quad u_{2}=x_{1}, \ldots, u_{d}=x_{d-1}
$$

so that $1-|u|=x_{d}$ and, using superscript for the variable with respect to which the derivative is taking,

$$
\partial_{1}^{(u)}=-\partial_{d}^{(x)}, \quad \partial_{2}^{(u)}=\partial_{1}^{(x)}-\partial_{d}^{(x)}, \ldots, \partial_{d}^{(u)}=\partial_{d-1}^{(x)}-\partial_{d}^{(x)},
$$

we obtain from the Rodrigue formula (2.9) that

$$
P_{\mathbf{n}}^{\boldsymbol{\gamma}_{S}}(u)=(-1)^{n_{1}} x_{S}^{-\gamma_{S}} x_{d}^{-\gamma_{d}} \frac{\partial^{|\mathbf{n}|}}{\partial_{d}^{n_{1}} \partial_{1, d}^{n_{2}} \cdots \partial_{d-1, d}^{n_{d}}}\left[x_{S}^{\gamma_{S}+\mathbf{n}} x_{d}^{\gamma_{d}+|\mathbf{n}|}\right]=P_{\mathbf{n}}^{\boldsymbol{\gamma}_{S}}\left(x_{S}\right) .
$$


Furthermore, under this change of variables, $W_{\gamma}(x)=W_{\gamma_{S}}(u)$. Consequently,

$$
\int_{T^{d}} P_{\mathbf{n}}^{\boldsymbol{\gamma}_{S}}\left(x_{S}\right) g(x) W_{\boldsymbol{\gamma}}(x) d x=\int_{T^{d}} P_{\mathbf{n}}^{\boldsymbol{\gamma}_{S}}(u) g\left(u_{2}, \ldots, u_{d}, 1-|u|\right) W_{\boldsymbol{\gamma}_{S}}(u) d u=0
$$

by the orthogonality of $P_{\mathbf{n}}^{\gamma}$, so that $P_{\mathbf{n}}^{\gamma_{S}}\left(x_{S}\right) \in \mathcal{V}_{n}^{d}\left(W_{\gamma}\right)$.

Another basis of $\mathcal{V}_{n}^{d}\left(W_{\boldsymbol{\gamma}}\right)$ is $\left\{V_{\mathbf{n}}^{\gamma}(x):|\mathbf{n}|=n, \mathbf{n} \in \mathbb{N}_{0}^{d}\right\}$, where $V_{\mathbf{n}}^{\gamma}$ are monomial orthogonal polynomials defined by

$$
V_{\mathbf{n}}^{\gamma}(x):=\sum_{\mathbf{m} \leq \mathbf{n}}(-1)^{n+|\mathbf{m}|} \prod_{i=1}^{d}\left(\begin{array}{c}
n_{i} \\
m_{i}
\end{array}\right) \frac{\left(\gamma_{i}+1\right)_{n_{i}}(|\gamma|+d)_{n+|\mathbf{m}|} \mid}{\left(\gamma_{i}+1\right)_{m_{i}}(|\boldsymbol{\gamma}|+d)_{n+|\mathbf{n}|}} x^{\mathbf{m}} .
$$

These polynomials satisfy $V_{\mathbf{n}}^{\boldsymbol{\gamma}}(x)=x^{\mathbf{n}}+Q_{\mathbf{n}}(x), Q_{\mathbf{n}} \in \Pi_{n-1}^{d}$ and $V_{\mathbf{n}}^{\boldsymbol{\gamma}} \in \mathcal{V}_{n}^{d}\left(W_{\boldsymbol{\gamma}}\right)$, so that $V_{\mathbf{n}}^{\gamma}$ is the orthogonal projection of $x^{\mathbf{n}}$ in $\mathcal{V}_{n}^{d}\left(W_{\boldsymbol{\gamma}}\right)$. Furthermore, we have the biorthogonal relation

$$
\int_{T^{d}} P_{\mathbf{n}}^{\gamma}(x) V_{\mathbf{m}}^{\gamma}(x) W_{\boldsymbol{\gamma}}(x) d x=\frac{\prod_{i=1}^{d}\left(\gamma_{i}+1\right)_{n_{i}}\left(\gamma_{d+1}+1\right)_{|\mathbf{n}|}}{(|\gamma|+d+1)_{2|\mathbf{n}|}} \mathbf{n} ! \delta_{\mathbf{n}, \mathbf{m}} .
$$

We note that $V_{\mathbf{n}}^{\gamma}$ is well defined even if $\gamma_{d+1}=-1$, since $\gamma_{d+1}$ appears only in $|\gamma|$ in the right hand side of (2.11). For latter use, we state one more property of $V_{\mathbf{n}}^{\gamma}$. Let $e_{1}, \ldots, e_{d}$ be the standard basis of $\mathbb{R}^{d}$, that is, the $i$ th coordinate of $e_{j}$ is 1 if $i=j, 0$ if $i \neq j$.

Lemma 2.6. For $1 \leq i \leq d$,

$$
\frac{\partial}{\partial x_{i}} V_{\mathbf{n}}^{\gamma}(x)=n_{i} V_{\mathbf{n}-e_{i}}^{\left(\gamma+e_{i}, \gamma_{d+1}+1\right)}(x) .
$$

Proof. We can assume $i=1$ and write $V_{\mathbf{n}}^{\gamma}$ as

$$
V_{\mathbf{n}}^{\boldsymbol{\gamma}}(x):=\sum_{m_{1}=0}^{n_{1}} \sum_{\mathbf{m}^{\prime} \leq \mathbf{n}^{\prime}}(-1)^{n+|\mathbf{m}|} \prod_{i=1}^{d}\left(\begin{array}{c}
n_{i} \\
m_{i}
\end{array}\right) \frac{\left(\gamma_{i}+1\right)_{n_{i}}(|\boldsymbol{\gamma}|+d)_{n+|\mathbf{m}|}}{\left(\gamma_{i}+1\right)_{m_{i}}(|\boldsymbol{\gamma}|+d)_{n+|\mathbf{n}|}} x_{1}^{m_{1}} \mathbf{x}^{\mathbf{m}^{\prime}}
$$

where $\mathbf{x}=\left(x_{2}, \ldots, x_{d}\right), \mathbf{n}^{\prime}=\left(n_{2}, \ldots, n_{d}\right)$ and $\mathbf{m}^{\prime}=\left(m_{2}, \ldots, m_{d}\right)$. Taking derivative with respect to $x_{1}$ and shifting the summation index over $m_{1}$, we obtain

$$
\begin{gathered}
\frac{\partial}{\partial x_{1}} V_{\mathbf{n}}^{\gamma}(x)=\sum_{m_{1}=1}^{n_{1}} \sum_{\mathbf{m}^{\prime} \leq \mathbf{n}^{\prime}}(-1)^{n+|\mathbf{m}|} \prod_{i=1}^{d}\left(\begin{array}{c}
n_{i} \\
m_{i}
\end{array}\right) \frac{\left(\gamma_{i}+1\right)_{n_{i}}(|\gamma|+d)_{n+|\mathbf{m}|}}{\left(\gamma_{i}+1\right)_{m_{i}}(|\gamma|+d)_{n+|\mathbf{n}|}} m_{1} x_{1}{ }^{m_{1}-1} \mathbf{x}^{\mathbf{m}^{\prime}} \\
=\sum_{m_{1}=0}^{n_{1}-1} \sum_{\mathbf{m}^{\prime} \leq \mathbf{n}^{\prime}}(-1)^{n+|\mathbf{m}|-1} n_{1}\left(\begin{array}{c}
n_{1}-1 \\
m_{1}
\end{array}\right) \frac{\left(\gamma_{1}+1\right)_{n_{1}}}{\left(\gamma_{1}+1\right)_{m_{1}+1}} x_{1} m_{1} \\
\times \prod_{i=2}^{d}\left(\begin{array}{c}
n_{i} \\
m_{i}
\end{array}\right) \frac{\left(\gamma_{i}+1\right)_{n_{i}}(|\gamma|+d)_{n+|\mathbf{m}|+1}}{\left(\gamma_{i}+1\right)_{m_{i}}(|\gamma|+d)_{n+|\mathbf{n}|}} \mathbf{x}^{\mathbf{m}^{\prime}} .
\end{gathered}
$$

Since $(a)_{n}=a(a+1)_{n-1}$, it holds that

$$
\frac{\left(\gamma_{1}+1\right)_{n_{1}}}{\left(\gamma_{1}+1\right)_{m_{1}+1}}=\frac{\left(\left(\gamma_{1}+1\right)+1\right)_{n_{1}-1}}{\left(\left(\gamma_{1}+1\right)+1\right)_{m_{1}}}, \quad \frac{(|\boldsymbol{\gamma}|+d)_{n+|\mathbf{m}|+1}}{(|\gamma|+d)_{n+|\mathbf{n}|}}=\frac{(|\gamma|+d+2)_{n+|\mathbf{m}|-1}}{(|\boldsymbol{\gamma}|+d+2)_{n+|\mathbf{n}|-2}}
$$

from which (2.12) follows. 
The relation (2.12) allows us to take higher order derivatives of $V_{\mathbf{n}}^{\boldsymbol{\gamma}}$. One interesting consequence of the lemma is that $V_{\mathbf{n}}^{\gamma}$ are orthogonal polynomials for a family of other inner products that involve differentiation. To state the result, we need further notation. For a subset $S=\left\{i_{1}, \ldots, i_{j}\right\}$ of $\mathbb{Z}_{d}$, we define

$$
\frac{\partial^{|S|}}{\partial x^{S}}=\frac{\partial^{|S|}}{\partial x_{i_{1}} \ldots \partial x_{i_{j}}}
$$

Lemma 2.7. Let $\gamma_{i}>-1$ for $1 \leq i \leq d+1$. For $m \leq d$, define

$$
[f, g]_{\boldsymbol{\gamma}}:=\langle f, g\rangle_{\boldsymbol{\gamma}}+\sum_{j=1}^{m} \sum_{\substack{S \subset \mathbb{Z}_{d} \\|S|=j}} \lambda_{S} \int_{T^{d}} \frac{\partial^{j} f}{\partial x^{S}} \frac{\partial^{j} g}{\partial x^{S}} \prod_{i \in S} x_{i}(1-|x|)^{j} W_{\boldsymbol{\gamma}}(x) d x,
$$

where $\lambda_{S}$ are nonnegative numbers. Then the polynomials $V_{\mathbf{n}}^{\boldsymbol{\gamma}}$ in (2.11) are orthogonal with respect to the inner product $[\cdot, \cdot]_{\boldsymbol{\gamma}}$.

Proof. By (2.12), taking repeated derivatives of $V_{\mathbf{n}}^{\gamma}$, we have

$$
\frac{\partial^{|S|} f}{\partial x^{S}} V_{\mathbf{n}}^{\gamma}(x)=n_{i_{1}} \cdots n_{i_{j}} V_{\mathbf{n}-e_{i_{1}}-\ldots-e_{i_{j}}}^{\left(\gamma+e_{i_{1}}+\ldots+e, \gamma_{d+1}+j\right)}(x)
$$

with $S=\left\{i_{1}, \ldots, i_{j}\right\}$, which implies, by the orthogonality of $V_{\mathbf{n}-e_{i_{1}}-\ldots-e_{i_{j}}}^{\left(\gamma+e_{i_{1}}+\ldots+e_{i_{j}}+j\right)}$, that $V_{\mathbf{n}}^{\gamma}$ is orthogonal with respect to

$$
[f, g]_{S}:=\int_{T^{d}} \frac{\partial^{j} f}{\partial x^{S}} \frac{\partial^{j} g}{\partial x^{S}} \prod_{i \in S} x_{i}(1-|x|)^{j} W_{\gamma}(x) d x
$$

and, consequently, orthogonal with respect to $[\cdot, \cdot]_{\boldsymbol{\gamma}}$. Since all $\lambda_{S} \geq 0,[\cdot, \cdot]_{\boldsymbol{\gamma}}$ is an inner product.

Remark 2.2. Using the relation (2.12) repeatedly, we can also include partial derivatives of higher orders, such as $\frac{\partial^{|\mathbf{m}|}}{\partial x_{1}^{m_{1}} \ldots \partial x_{k}^{m_{k}}}$ for $\mathbf{m}=\left(m_{1}, \ldots, m_{k}\right)$ with $k \leq d$, in the definition of $[f, g]_{\boldsymbol{\gamma}}$. For our purpose, the definition in (2.13) is sufficient.

A couple of remarks are in order. We note that $[\cdot, \cdot]_{S}$ is not an inner product by itself since, for example, $[f, f]_{S}=0$ for $f(x)=1$. Even though $\left\{V_{\mathbf{n}}^{\boldsymbol{\gamma}}:|\mathbf{n}|=n\right\}$ is a basis of $\mathcal{V}_{n}^{d}\left(W_{\boldsymbol{\gamma}}\right)$, not every basis of $\mathcal{V}_{n}^{d}\left(W_{\boldsymbol{\gamma}}\right)$ is orthogonal with respect to $[\cdot, \cdot]_{\boldsymbol{\gamma}}$. For example, when $\lambda_{S}$ are not all zero, $P_{\mathbf{n}}^{\gamma}$ is not an orthogonal polynomial with respect to $[\cdot, \cdot]_{\boldsymbol{\gamma}}$.

2.4. Orthogonal polynomials on the faces of the simplex. In addition to the standard basis $e_{1}, \ldots, e_{d}$ of $\mathbb{R}^{d}$, we further set $e_{0}=(0, \ldots, 0)$. The points $e_{0}, e_{1}, \ldots, e_{d}$ are the vertices of the simplex $T^{d}$. The boundary of the simplex $T^{d}$ can be decomposed into lower dimensional faces.

Definition 2.8. Let $S$ be a subset of $\mathbb{Z}_{d+1}$ with $1 \leq|S| \leq d$. Then

$$
T_{S}^{d-|S|}:=\left\{x \in T^{d}: x_{i}=0, i \in S\right\}
$$

is a $(d-|S|)$-dimensional face of $T^{d}$.

For $1 \leq|S| \leq d$, the number of $(d-|S|)$-dimensional faces is $\left(\begin{array}{c}d+1 \\ |S|\end{array}\right)$. In particular, for $|S|=d$, the 0 -dimensional faces are precisely the vertices $e_{0}, e_{1}, \ldots, e_{d}$ of $T^{d}$, whereas for $|S|=1$, the $(d-1)$-dimensional faces are $T_{\{j\}}^{d-1}, j=1, \ldots, d, d+1$. Each 
face $T_{S}^{d-|S|}$ is a $(d-|S|)$-dimensional simplex and, moreover, $T_{S}^{d-|S|}$ is isomorphic to the simplex $T^{d-|S|}$ in variables $\left\{x_{i}: i \in S^{c}\right\}$. Furthermore, the restriction of $W_{\gamma}$ on $T_{S}^{d-|S|}$ is a weight function of the same type in variables $\left\{x_{i}: i \in S^{c}\right\}$.

Definition 2.9. Let $S=\left\{i_{1}, \ldots, i_{d}\right\}$ be a subset of $\mathbb{Z}_{d+1}$ and $S^{c}=\left\{i_{d+1}\right\}$. Let $\gamma_{S}:=\left(\gamma_{S}, \gamma_{i_{d+1}}\right)$, where $\gamma_{S}:=\left(\gamma_{i_{1}}, \ldots, \gamma_{i_{d}}\right)$, and $x_{S}=\left(x_{i_{1}}, \ldots, x_{i_{d}}\right)$. Let $P_{\mathbf{n}}^{\gamma_{S}}\left(x_{S}\right)$ be defined as Definition 2.4.

1. Let $d+1 \notin S$. For $S_{j} \subset \mathbb{Z}_{d}$ with $\left|S_{j}\right|=j, 1 \leq j \leq d-1$, define

$$
H_{n, S_{j}}^{d}\left(W_{\gamma}\right):=\operatorname{span}\left\{P_{\mathbf{n}}^{\gamma}(x):|\mathbf{n}|=n, \text { and } n_{\ell}=0, \forall \ell \in S_{j}\right\} .
$$

2. Let $d+1 \in S$. Then $S=\left\{d+1, \ell_{1}, \ldots, \ell_{d-1}\right\}$ with $\left\{\ell_{1}, \ldots, \ell_{d-1}\right\} \subset \mathbb{Z}_{d}$ and $\mathbb{Z}_{d+1} \backslash S=\left\{\ell_{d}\right\}$. For $S_{j}=\left\{d+1, \ell_{1}, \ldots, \ell_{j-1}\right\}$ with $1 \leq j \leq d-1$, where $S_{1}=\{d+1\}$, define

$$
H_{n, S_{j}}^{d}\left(W_{\gamma}\right):=\operatorname{span}\left\{P_{\mathbf{n}}^{\left(\gamma_{S}, \gamma_{\ell_{d}}\right)}\left(x_{S_{j}}, x_{S \backslash S_{j}}\right):|\mathbf{n}|=n \text { with } n_{\ell}=0,1 \leq \ell \leq j\right\} .
$$

We further define $H_{n, S_{d}}^{d}\left(W_{\gamma}\right)=\emptyset$ and $H_{n, S_{d+1}}^{d}\left(W_{\gamma}\right)=\emptyset$.

Lemma 2.10. The space $H_{n, S_{j}}^{d}\left(W_{\gamma}\right)$ satisfies the following properties:

(i) $H_{n, S_{j}}^{d}\left(W_{\gamma}\right)$ is a subspace of $\mathcal{V}_{n}^{d}\left(W_{\gamma}\right)$;

(ii) The restriction of $H_{n, S_{j}}^{d}\left(W_{\gamma}\right)$ on the face $T_{S_{j}}^{d-j}$ is the subspace $\mathcal{V}_{n}^{d-j}\left(\left.W_{\gamma}\right|_{T_{S_{j}}^{d-j}}\right)$, where $\left.W_{\gamma}\right|_{T_{S_{j}}^{d-j}}$ denotes the restriction of $W_{\gamma}$ on the face $T_{S_{j}}^{d-j}$, that is,

$$
\left.H_{n, S_{j}}^{d}\left(W_{\gamma}\right)\right|_{T_{S_{j}}^{d-j}}=\mathcal{V}_{n}^{d-j}\left(\left.W_{\gamma}\right|_{T_{S_{j}}^{d-j}}\right)
$$

in the variables $\left\{x_{i}: i \in S_{j}^{c}\right\}$.

Proof. The item (i) follows immediately from Lemma 2.5. For the proof of item (ii), we need to consider two cases, $d+1 \notin S$ and $d+1 \in S$.

Case 1. $d+1 \notin S$. Then $S=\mathbb{Z}_{d}$. Let $S_{j}=\left\{i_{1}, \ldots, i_{j}\right\} \subset \mathbb{Z}_{d}, S_{j}^{c}=\left\{i_{j+1}, \ldots, i_{d}\right\}$, $\gamma_{S_{j}^{c}}=\left(\gamma_{i_{j+1}}, \ldots, \gamma_{i_{d}}\right)$ and $\mathbf{n}_{S_{j}^{c}}=\left(n_{i_{j+1}}, \ldots, n_{i_{d}}\right)$. If $n_{\ell}=0$ for $\ell \in S_{j}$, then $P_{\mathbf{n}}^{\gamma}$ in (2.9) can be written as

$$
P_{\mathbf{n}}^{\gamma}(x)=x_{S_{j}^{c}}^{-\gamma_{S_{j}^{c}}}(1-|x|)^{-\gamma_{d+1}} \frac{\partial^{\left|\mathbf{n}_{S_{j}^{c}}\right|}}{\partial x_{S_{j}^{c}}^{\mathbf{n}_{S_{j}^{c}}^{c}}}\left[x_{S_{j}^{c}}^{\gamma_{S_{j}^{c}+\mathbf{n}_{S_{j}^{c}}}}(1-|x|)^{\gamma_{d+1}+\left|\mathbf{n}_{S_{j}^{c} \mid}\right|}\right],
$$

which, when restricted to the face $T_{S_{j}}^{d-j}$, becomes

$$
\left.P_{\mathbf{n}}^{\gamma}(x)\right|_{T_{S_{j}}^{d-j}}=x_{S_{j}^{c}}^{-\gamma_{S_{j}^{c}}}\left(1-\left|x_{S_{j}^{c}}\right|\right)^{-\gamma_{d+1}} \frac{\partial^{\left|\mathbf{n}_{S_{j}^{c}}\right|}}{\partial x_{S_{j}^{c}}^{\mathbf{n}_{S_{j}^{c}}}}\left[x_{S_{j}^{c}}^{\gamma_{S_{j}^{c}+\mathbf{n}_{S_{j}^{c}}^{c}}}\left(1-\left|x_{S_{j}^{c}}\right|\right)^{\gamma_{d+1}+\left|\mathbf{n}_{S_{j}^{c}}^{c}\right|}\right],
$$

precisely the Rodrigue formula in the variable $x_{S_{j}^{c}}$ for the weight function $\left.W_{\gamma}\right|_{T_{S_{j}}^{d-j}}$ and, as such, is an element of $\mathcal{V}_{n}^{d-j}\left(\left.W_{\gamma}\right|_{T_{S_{j}}^{d-j}}\right)$. Furthermore, the collection of such polynomials forms a basis of the latter space. This completes the proof of (ii) in this case. 
Case 2. $d+1 \in S$. Up to a permutation in $\mathcal{G}_{d}$, we can assume $S=(d+1,1, \ldots, d-1)$. Then $S^{c}=\{d\}, \gamma_{S}=\left(\gamma_{S}, \gamma_{d}\right)$ with $\gamma_{S}=\left(\gamma_{d+1}, \gamma_{1}, \ldots, \gamma_{d-1}\right)$ and $S_{j}=\{d+$ $1,1, \ldots, j-1\}$. Making a change of variable

$$
u_{1}=x_{d+1}=1-|x|, \quad u_{2}=x_{1}, \ldots, u_{d}=x_{d-1},
$$

we have $1-|u|=x_{d}, W_{\gamma}(x)=W_{\gamma_{S}}(u)$ and $P_{\mathbf{n}}^{\gamma_{S}}\left(x_{S}\right)=P_{\mathbf{n}}^{\gamma_{S}}(u)$, as in the proof of Lemma 2.5, so that

$$
H_{n, S_{j}}^{d}\left(W_{\gamma}(x)\right)=H_{n, S_{j}^{*}}^{d}\left(W_{\gamma_{S}}(u)\right)
$$

where $S_{j}^{*}:=\{1,2, \ldots, j\}$ and

$$
H_{n, S_{j}^{*}}^{d}\left(W_{\boldsymbol{\gamma}_{S}}(u)\right)=\operatorname{span}\left\{P_{\mathbf{n}}^{\gamma_{S}}(u):|\mathbf{n}|=n, n_{\ell}=0, \forall \ell \in S_{j}^{*}\right\} .
$$

From the case that $d+1 \notin S$, it follows that

$$
\left.H_{n, S_{j}^{*}}^{d}\left(W_{\gamma_{S}}\right)\right|_{T_{S_{j}^{*}}^{d-j}}=\mathcal{V}_{n}^{d-j}\left(\left.W_{\gamma_{S}}\right|_{T_{S_{j}^{* j}}^{d-j}}\right),
$$

which is equivalent to

$$
\left.H_{n, S_{j}}^{d}\left(W_{\gamma}\right)\right|_{T_{S_{j}}^{d-j}}=\mathcal{V}_{n}^{d-j}\left(\left.W_{\gamma}\right|_{T_{S_{j}}^{d-j}}\right)
$$

This completes the proof.

Lemma 2.11. Let $H_{n, S_{j}}^{d}\left(W_{\gamma}\right)$ be defined as in Definition 2.9. Then $H_{n, S_{j}}^{d}\left(W_{\gamma}\right)$ is a subspace of $\mathcal{V}_{n}^{d}\left(W_{\boldsymbol{\gamma}^{*}}\right)$, where $\boldsymbol{\gamma}^{*}=\left(\gamma_{1}^{*}, \ldots, \gamma_{d+1}^{*}\right)$ is defined as follows: $\gamma_{\ell}^{*} \in \mathbb{R}$ are arbitrary for $\ell \in S_{j}$ and $\gamma_{\ell}^{*}=\gamma_{j}$ otherwise. In particular, every element of $H_{n, S_{j}}^{d}\left(W_{\gamma}\right)$ satisfies the equation $L_{\boldsymbol{\gamma}^{*}} P=-n\left(n+\left|\gamma^{*}\right|+d\right) P$, where $\gamma_{\ell}^{*}$ are arbitrary for $\ell \in S_{j}$.

Proof. In the Rodrigue formula $P_{\mathbf{n}}^{\gamma}$ of (2.9), if $n_{\ell}=0$, then the derivative with respect to $x_{\ell}$ does not appear, so that, after canceling out $x_{\ell}^{-\gamma_{\ell}}$ and $x_{\ell}^{\gamma_{\ell}}, \gamma_{\ell}$ does not appear in $P_{\mathbf{n}}^{\gamma}$, which means that we can assign $\gamma_{\ell}$ any value. In other words, $P_{\mathbf{n}}^{\gamma}(x)=P_{\mathbf{n}}^{\gamma^{*}}(x)$ if $n_{\ell}=0, \ell \in S_{j}$. Consequently, $H_{n, S_{j}}^{d}\left(W_{\gamma}\right) \in \mathcal{V}_{n}^{d}\left(W_{\gamma}^{*}\right)$.

\section{MAin Results}

Our main results on the simplex are fairly involved in notations. We shall state our results first in the case of triangle, that is, $d=2$, and use it to explain how we arrive at the results.

3.1. Sobolev orthogonal polynomials on the triangle. As stated in the introduction, we are facing two problems. The first one is to find a complete solutions of polynomials for the differential equation (1.2) in which $d=2$ and $\gamma=(\alpha, \beta, \gamma)$.

To facilitate the discussion, we define the following subspaces of $\mathcal{V}_{n}^{2}\left(W_{\alpha, \beta, \gamma}\right)$,

$$
\begin{aligned}
& H_{n, 1}\left(w_{\gamma, \beta}\right):=\operatorname{span}\left\{P_{0, n}^{(0, \beta, \gamma)}(x, y)\right\}, \quad H_{n, 2}\left(w_{\gamma, \alpha}\right):=\operatorname{span}\left\{P_{0, n}^{(0, \alpha, \gamma)}(y, x)\right\}, \\
& H_{n, 3}\left(w_{\alpha, \beta}\right):=\operatorname{span}\left\{P_{0, n}^{(0, \beta, \alpha)}(1-x-y, y)\right\},
\end{aligned}
$$

which can be formulated as special cases of the spaces in Definition 2.9. According to Proposition 2.1 $\left.H_{n, 1}\left(w_{\gamma, \beta}\right)\right|_{x=0}=\mathcal{V}_{n}^{1}\left(w_{\gamma, \beta}\right),\left.H_{n, 2}\left(w_{\gamma, \alpha}\right)\right|_{y=0}=\mathcal{V}_{n}^{1}\left(w_{\gamma, \alpha}\right)$ and $\left.H_{n, 3}\left(w_{\alpha, \beta}\right)\right|_{x+y=1}=\mathcal{V}_{n}^{1}\left(w_{\alpha, \beta}\right)$. In the following we adopt the convention that

$$
\mathcal{V}_{n}^{2}\left(W_{\alpha, \beta, \gamma}\right)=\emptyset \quad \text { and } \quad H_{n, j}=\emptyset, \quad \text { if } n<0 .
$$


Theorem 3.1. For $n \in \mathbb{N}_{0}$ and at least one of $\alpha, \beta, \gamma$ being -1 , the differential equation

$$
L_{\alpha, \beta, \gamma} u=-n(n+\alpha+\beta+\gamma+2) u
$$

has a solution space $\mathcal{U}_{n}^{2}\left(W_{\alpha, \beta, \gamma}\right)$ of polynomials of degree $n$, which has dimension $n+1$ and can be decomposed as a direct sum as follows:

$$
\begin{aligned}
\mathcal{U}_{n}^{2}\left(W_{\alpha, \beta,-1}\right):= & (1-x-y) \mathcal{V}_{n-1}^{2}\left(W_{\alpha, \beta, 1}\right)+H_{n, 3}\left(w_{\alpha, \beta}\right), \quad n \geq 0 \\
\mathcal{U}_{n}^{2}\left(W_{\alpha,-1,-1}\right):= & y(1-x-y) \mathcal{V}_{n-2}^{2}\left(W_{\alpha, 1,1}\right)+y H_{n-1,3}\left(w_{\alpha, 1}\right) \\
& +(1-x-y) H_{n-1,2}\left(w_{1, \alpha}\right)
\end{aligned}
$$

for $n \geq 1$ and $\mathcal{U}_{0}^{2}\left(W_{\alpha,-1,-1}\right):=\operatorname{span}\{1\}$, and

$$
\begin{aligned}
\mathcal{U}_{n}^{2}\left(W_{-1,-1,-1}\right):= & x y(1-x-y) \mathcal{V}_{n-3}^{2}\left(W_{1,1,1}\right)+x y H_{n-2,3}\left(w_{1,1}\right) \\
& +x(1-x-y) H_{n-2,2}\left(w_{1,1}\right)+y(1-x-y) H_{n-2,1}\left(w_{1,1}\right)
\end{aligned}
$$

for $n \geq 2, \mathcal{U}_{0}^{2}\left(W_{-1,-1,-1}\right):=\operatorname{span}\{1\}$ and $\mathcal{U}_{1}^{2}\left(W_{-1,-1,-1}\right):=\operatorname{span}\left\{x+c_{1}, y+c_{2}\right\}$, where $c_{1}$ and $c_{2}$ are arbitrary constants.

From the decomposition, we can deduce further relations among the subspaces.

Corollary 3.2. The subspaces in the Theorem 3.1 satisfy the relations

$$
\begin{aligned}
\mathcal{U}_{n}^{2}\left(W_{\alpha,-1,-1}\right) & =y \mathcal{U}_{n-1}^{2}\left(W_{\alpha, 1,-1}\right)+(1-x-y) H_{n-1,2}\left(w_{1, \alpha}\right), \quad n \geq 1 \\
\mathcal{U}_{n}^{2}\left(W_{-1,-1,-1}\right) & =x \mathcal{U}_{n-1}^{2}\left(W_{1,-1,-1}\right)+y(1-x-y) H_{n-2,1}\left(w_{1,1}\right), \quad n \geq 2 .
\end{aligned}
$$

Remark 3.1. In the statement of the theorem, we could also assume either $\alpha=-1$ or $\beta=-1$ instead of $\gamma=-1$. The result then can be obtained via permutation of $(\alpha, \beta, \gamma)$ and the corresponding permutation of $(x, y, 1-x-y)$. We give one example explicitly:

$$
\mathcal{U}_{n}^{2}\left(W_{-1,-1, \gamma}\right)=x y \mathcal{V}_{n-2}^{2}\left(W_{1,1, \gamma}\right)+x H_{n-1,2}\left(w_{\gamma, 1}\right)+y H_{n-1,1}\left(w_{\gamma, 1}\right) .
$$

Our second problem is to identify an inner product under which the eigenspaces in the Theorem 3.1 are orthogonal.

Definition 3.3. Let $\lambda_{i}$ and $\lambda_{i, j}$ be nonnegative numbers. Define the bilinear forms

$$
\begin{aligned}
\langle f, g\rangle_{\alpha, \beta,-1}:= & \int_{T^{2}}\left[x \partial_{x} f(x, y) \partial_{x} g(x, y)+y \partial_{y} f(x, y) \partial_{y} g(x, y)\right] x^{\alpha} y^{\beta} d x d y \\
& +\lambda_{1} \int_{0}^{1} f(x, 1-x) g(x, 1-x) x^{\alpha}(1-x)^{\beta} d x \\
\langle f, g\rangle_{\alpha,-1,-1}:= & \int_{T^{2}} \partial_{y} f(x, y) \partial_{y} g(x, y) x^{\alpha} d x d y, \\
& +\lambda_{1} \int_{0}^{1} \partial_{x} f(x, 0) \partial_{x} g(x, 0) x^{\alpha+1} d x+\lambda_{1,0} f(1,0) g(1,0) \\
\langle f, g\rangle_{-1,-1,-1}:= & \int_{T^{2}} \partial_{x y} f(x, y) \partial_{x y} g(x, y)(1-x-y) d x d y, \\
& +\lambda_{1} \int_{0}^{1} \partial_{x} f(x, 0) \partial_{x} g(x, 0) d x+\lambda_{2} \int_{0}^{1} \partial_{y} f(0, y) \partial_{y} g(0, y) d y \\
& +\lambda_{1,0} f(1,0) g(1,0)+\lambda_{0,1} f(0,1) g(0,1)+\lambda_{0,0} f(0,0) g(0,0) .
\end{aligned}
$$

Theorem 3.4. Let $\alpha, \beta>-1$. For $n=0,1,2, \ldots$, the following holds: 
(1) If $\lambda_{1}>0$ then $\langle f, g\rangle_{\alpha, \beta,-1}$ is an inner product for which $\mathcal{U}_{n}^{2}\left(W_{\alpha, \beta,-1}\right)$ is the space of orthogonal polynomials of degree $n$;

(2) If $\lambda_{1}>0$ and $\lambda_{1,0}>0$, then $\langle f, g\rangle_{\alpha,-1,-1}$ is an inner product for which $\mathcal{U}_{n}^{2}\left(W_{\alpha,-1,-1}\right)$ is the space of orthogonal polynomials of degree $n$;

(3) If $\lambda_{1}>0, \lambda_{2}>0$ and at least one of $\lambda_{1,0}, \lambda_{0,1}, \lambda_{0,0}$ is positive, then $\langle f, g\rangle_{-1,-1,-1}$ is an inner product for which $\mathcal{U}_{n}^{2}\left(W_{-1,-1,-1}\right)$ is the space of orthogonal polynomials of degree $n$, where we redefine $\mathcal{U}_{1}^{2}\left(W_{-1,-1,-1}\right)$ as

$$
\mathcal{U}_{1}^{2}\left(W_{-1,-1,-1}\right)=\operatorname{span}\left\{x-\frac{\lambda_{1,0}}{\lambda_{1,0}+\lambda_{0,1}+\lambda_{0,0}}, y-\frac{\lambda_{0,1}}{\lambda_{1,0}+\lambda_{0,1}+\lambda_{0,0}}\right\} .
$$

With respect to $\langle\cdot, \cdot\rangle_{\alpha, \beta,-1}$, the decomposition $\mathcal{U}_{n}^{2}\left(W_{\alpha, \beta,-1}\right)$ is an orthogonal one, that is,

$$
\mathcal{U}_{n}^{2}\left(W_{\alpha, \beta,-1}\right)=(1-x-y) \mathcal{V}_{n-1}^{2}\left(W_{\alpha, \beta, 1}\right) \bigoplus H_{n, 3}\left(w_{\alpha, \beta}\right)
$$

as will be shown in (iii) of Theorem 3.7 .

Again, by symmetry, we can state the inner product if other parameters are -1 . We give one example.

$$
\begin{aligned}
\langle f, g\rangle_{-1,-1, \gamma}:= & \int_{T^{2}}\left(\partial_{y}-\partial_{x}\right) f(x, y)\left(\partial_{y}-\partial_{x}\right) g(x, y)(1-x-y)^{\gamma} d x d y \\
& +\lambda_{1} \int_{0}^{1} \partial_{x} f(x, 0) \partial_{x} g(x, 0)(1-x)^{\gamma+1} d x \\
& +\lambda_{2} \int_{0}^{1} \partial_{y} f(0, y) \partial_{y} g(0, y)(1-y)^{\gamma+1} d y+\lambda_{0,0} f(0,0) g(0,0)
\end{aligned}
$$

where $\lambda_{1}, \lambda_{2} \geq 0$ with at least one of them positive and $\lambda_{0,0}>0$.

Remark 3.2. Notice that $\langle f, g\rangle_{-1,-1, \gamma}$ contains two terms of one-dimenional integral, whereas $\langle f, g\rangle_{\alpha,-1,-1}$ contains only one such term. Evidently, by symmetry we could include another term in $\langle f, g\rangle_{\alpha,-1,-1}$. In fact, it will become clear in the discussion below that we could include even more terms if we are willing to use higher order derivatives. Apart from consideration of symmetry, as in the three vertices in $\langle f, g\rangle_{-1 .-1 .-1}$ in Definition 3.3. we shall keep the number of terms in the inner product minimal below.

These theorems will be special cases of the results on $T^{d}$. We will not give separate proof for $d=2$. Instead we give an indication below on how the main terms of the inner products are identitified. We start from an observation in 6 that the monic orthogonal polynomials $V_{k, n}^{(\alpha, \beta, \gamma)}(x, y)$ are also orthogonal with respect to the inner product, when $\alpha, \beta, \gamma>-1$,

$$
[f, g]_{\alpha, \beta, \gamma}=\langle f, g\rangle_{\alpha, \beta, \gamma}+\lambda_{1}\left\langle\partial_{x} f, \partial_{x} g\right\rangle_{\alpha+1, \beta, \gamma+1}+\lambda_{2}\left\langle\partial_{y} f, \partial_{y} g\right\rangle_{\alpha, \beta+1, \gamma+1},
$$

which is a special case of Lemma 2.7. Since $V_{k, n}^{\alpha, \beta, \gamma}$ are well-defined if $\gamma=-1$, letting $\gamma \mapsto-1$ preserves the orthogonality, which is how the orthogonality in $\langle f, g\rangle_{\alpha, \beta,-1}$ was established [6], but the method does not give the decomposition of the orthogonal space. Indeed, the second and the third terms in the right hand side of $[f, g]_{\alpha, \beta, \gamma}$ make sense for $\gamma=-1$ as well, whereas for the first term we parametrize the integral over $T^{2}$ as

$$
\int_{T^{2}} f(x, y) d x d y=\int_{0}^{1} \int_{0}^{1-y} f(x, y) d x d y
$$


and apply the limit relation

$$
\lim _{\mu \rightarrow-1^{+}} \frac{1}{\mu+1} \int_{0}^{1} f(x) x^{\mu} d x=f(0)
$$

to conclude that

$$
\begin{aligned}
\lim _{\gamma \rightarrow-1^{+}}[f, g]_{\alpha, \beta, \gamma}= & c_{\alpha, \beta} \int_{0}^{1} f(x, 1-x) g(x, 1-x) x^{\alpha}(1-x)^{\beta} d x \\
& +\lambda_{1}\left\langle\partial_{x} f, \partial_{x} g\right\rangle_{\alpha+1, \beta, 0}+\lambda_{2}\left\langle\partial_{y} f, \partial_{y} g\right\rangle_{\alpha, \beta+1,0}
\end{aligned}
$$

which is a constant multiple of $\langle f, g\rangle_{\alpha, \beta,-1}$ when $\lambda_{2}=\lambda_{1}$. Since $V_{k, n}^{(\alpha, \beta,-1)}(x)$ are well defined for all $0 \leq k \leq n$, their orthogonality is preserved under the above limit.

The above limit process can be continued one more time by taking, for example, $\beta \rightarrow-1$, which gives

$$
\lim _{\beta, \gamma \rightarrow-1^{+}}[f, g]_{\alpha, \beta, \gamma}=c\langle f, g\rangle_{\alpha,-1,-1},
$$

where $c$ is a constant. However, since $V_{k, n}^{(\alpha,-1,-1)}(x)$ are not well defined when $k=0$, the limit process at this stage no longer gives the complete answer for our problem.

If we take the limit $\alpha \rightarrow-1$ in $\langle f, g\rangle_{\alpha,-1,-1}$, we end up with

$$
\begin{aligned}
\lim _{\alpha \rightarrow-1^{+}}\langle f, g\rangle_{\alpha,-1,-1}= & \lambda_{1} \int_{0}^{1} \partial_{x} f(x, 0) \partial_{x} g(x, 0) d x+ \\
& +\lambda_{2} \int_{0}^{1} \partial_{y} f(0, y) \partial_{y} g(0, y) d y+\lambda_{1,0} f(1,0) g(1,0)
\end{aligned}
$$

which however is no longer an inner product since, for example, it is equal to zero if $f(x, y)=g(x, y)=x y$. In order to obtain the main terms of the inner product in $\langle f, g\rangle_{-1,-1,-1}$ we need to go one level up by noticing that, by Lemma $2.7 V_{k, n}^{(\alpha, \beta, \gamma)}$ are orthogonal with respect to yet one more inner product: For $\alpha, \beta, \gamma>-1$,

$$
\begin{aligned}
{[f, g]_{\alpha, \beta, \gamma}=} & \langle f, g\rangle_{\alpha, \beta, \gamma}+\lambda_{1}\left\langle\partial_{x} f, \partial_{x} g\right\rangle_{\alpha+1, \beta, \gamma+1} \\
& +\lambda_{2}\left\langle\partial_{y} f, \partial_{y} g\right\rangle_{\alpha, \beta+1, \gamma+1}+\lambda_{1,1}\left\langle\partial_{x y} f, \partial_{x y} g\right\rangle_{\alpha+1, \beta+1, \gamma+2}
\end{aligned}
$$

where $\lambda_{1}, \lambda_{2}, \lambda_{1,1}$ are nonnegative numbers. Taking the limit three times in the above inner product leads to the main terms of the inner product $\langle f, g\rangle_{-1,-1,-1}$.

We emphasis, however, that the above limiting process only suggests possible form of the Sobolev inner product. It neither gives the final answer nor it proves the orthogonality.

3.2. Sobolev orthogonal polynomials on the simplex. For a fixed positive integer $k, 1 \leq k \leq d$, we consider $W_{\gamma}$ with $\gamma_{d+2-k}=\ldots=\gamma_{d+1}=-1$. For convenience, we introduce the notation

$$
W_{\gamma,-\mathbf{1}_{k}}(x)=\left.W_{\gamma}(x)\right|_{\gamma_{d+2-k}=\ldots=\gamma_{d+1}=-1},
$$

where $\mathbf{1}_{k}:=(1, \ldots, 1) \in \mathbb{Z}^{k}$ and $-\mathbf{1}_{k}=(-1, \ldots,-1)$, and we always assume that the subindex of $W_{\gamma}$ is a vector in $\mathbb{R}^{d+1}$ so that, with the length of the vector $-\mathbf{1}_{k}$ being $k$, the length of $\gamma$ is $d-k+1, \gamma=\left(\gamma_{1}, \ldots, \gamma_{d-k+1}\right)$. In particular,

$$
W_{\gamma,-\mathbf{1}_{k}}(x)=x_{1}^{\gamma_{1}} \ldots x_{d-k+1}^{\gamma_{d-k+1}} x_{d-k+2}^{-1} \ldots x_{d}^{-1}(1-|x|)^{-1} \text {. }
$$


In these notations we can allow $k=d+1$ if we regard $\gamma \in \mathbb{R}^{d-k+1}$ as nonentity when $k=d+1$ and write $W_{-\mathbf{1}_{d+1}}(x)=W_{-\mathbf{1}}(x)$.

Recall the subspace $H_{n, S_{j}}^{d}\left(W_{\gamma}\right)$ in the Definition 2.9, From Remark 2.11, since the values of $\gamma_{i}$ for $i \in S_{j}$ can be arbitraty in the basis of $H_{n, S_{j}}^{d}\left(W_{\gamma}\right)$, we can set $\left\{\gamma_{i}=0, i \in S_{j}\right\}$. Let

$$
S_{k, j}(\sigma):=\left\{\ell: \quad \varepsilon_{\ell}=0,\left(\varepsilon_{1}, \ldots, \varepsilon_{d+1}\right)=\left(\gamma,\left(\mathbf{0}_{k-j}, \mathbf{1}_{j}\right) \sigma\right)\right\},
$$

so that $S_{k, j}(\sigma)$ contains the indices of zero elements in $\left(\mathbf{0}_{k-j}, \mathbf{1}_{j}\right) \sigma$, and we treat $S_{k, j}(\sigma)$ as a vector whose elements are arranged according to their order in $\left.\left(\gamma,\left(\mathbf{0}_{k-j}, \mathbf{1}_{j}\right) \sigma\right)\right\}$. To simplify the notation, we define for $j=0,1, \ldots, k-1$,

$$
H_{n}^{d}\left(W_{\gamma,\left(\mathbf{o}_{k-j}, \mathbf{1}_{j}\right) \sigma}\right):=H_{n, S_{k, j}(\sigma)}^{d}\left(W_{\gamma,\left(\mathbf{o}_{k-j}, \mathbf{1}_{j}\right) \sigma}\right),
$$

where if $\sigma=i d$, then $S_{k, 0}=\{d+2-k, \ldots, d+1\}$ and $\mathbf{1}_{0}$ is a nonentity. It then follows by Lemma 2.10 that

(i) $H_{n}^{d}\left(W_{\gamma,\left(\mathbf{0}_{k-j}, \mathbf{1}_{j}\right) \sigma}\right)$ is a subspace of $\mathcal{V}_{n}^{d}\left(W_{\gamma,\left(\mathbf{0}_{k-j}, \mathbf{1}_{j}\right) \sigma}\right)$;

(ii) The restriction of $H_{n}^{d}\left(W_{\gamma,\left(\mathbf{0}_{k-j}, \mathbf{1}_{j}\right) \sigma}\right)$ on the face $T_{S_{k, j}(\sigma)}^{d-(k-j)}$ agrees with the space $\mathcal{V}_{n}^{d-(k-j)}\left(\left.W_{\gamma,\left(\mathbf{0}_{k-j}, \mathbf{1}_{j}\right) \sigma}\right|_{T_{S_{k, j}(\sigma)}^{d-(k-j)}}\right)$ with variables in $\left\{x_{i}: i \in\left(S_{k, j}(\sigma)\right)^{c}\right\}$.

In particular, if $\sigma=i d$, then $S_{k, j}=\{d-k+2, \ldots, d+1-j\}$ and

$$
\left.H_{n}^{d}\left(W_{\gamma,\left(\mathbf{o}_{k-j}, \mathbf{1}_{j}\right)}\right)\right|_{T_{S_{k, j}^{d-(k-j)}}^{d-(k)}}=\mathcal{V}_{n}^{d-(k-j)}\left(\left.W_{\left(\gamma, \mathbf{0}_{k-j}, \mathbf{1}_{j}\right)}\right|_{T_{S_{k, j}}^{d-(k-j)}}\right)
$$

with variables $x_{1}, \ldots, x_{d-k+1}, x_{d+2-j}, \ldots, x_{d}$. Furthermore, by definition, we also have $H_{n}^{d}\left(W_{\gamma, \mathbf{0}_{k}}\right)=\emptyset$ if $k=d$ or $d+1$.

We adopt the convention that $\sum_{i=j}^{k} a_{i}=0$ if $k<j$ regardless the values of $a_{i}$.

Theorem 3.5. Let $k$ be a fixed integer, $1 \leq k \leq d+1$ and $\gamma \in \mathbb{R}^{d-k+1}$. For $n \in \mathbb{N}_{0}$, the differential equation

$$
L_{\gamma,-\mathbf{1}_{k}} u=-n(n+|\gamma|+d-k) u
$$

has a solution space $\mathcal{U}_{n}^{d}\left(W_{\gamma,-\mathbf{1}_{k}}\right)$ of polynomials of degree $n$ with

$$
\operatorname{dim} \mathcal{U}_{n}^{d}\left(W_{\gamma,-\mathbf{1}_{k}}\right)=\left(\begin{array}{c}
n+d-1 \\
n
\end{array}\right),
$$

which can be decomposed as a direct sum as follows:

(i) if $k=1$, then $\mathcal{U}_{0}^{d}\left(W_{\gamma,-1}\right)=\operatorname{span}\{1\}$ and for $n \geq 1$,

$$
\mathcal{U}_{n}^{d}\left(W_{\gamma,-1}\right)=x_{d+1} \mathcal{V}_{n-1}^{d}\left(W_{\gamma, 1}\right)+H_{n}^{d}\left(W_{\gamma, 0}\right) .
$$

(ii) if $2 \leq k \leq d$, then $\mathcal{U}_{0}^{d}\left(W_{\gamma,-\mathbf{1}_{k}}\right)=\operatorname{span}\{1\}$ and for $n \geq 1$,

$$
\begin{aligned}
\mathcal{U}_{n}^{d}\left(W_{\gamma,-\mathbf{1}_{k}}\right)= & x_{d+1-(k-1)} \cdots x_{d+1} \mathcal{V}_{n-k}^{d}\left(W_{\gamma, \mathbf{1}_{k}}\right) \\
& +\sum_{j=1}^{k-1} \sum_{\sigma \in \mathcal{G}_{k}} x_{j, k}(\sigma) H_{n-j}^{d}\left(W_{\gamma,\left(\mathbf{0}_{k-j}, \mathbf{1}_{j}\right) \sigma}\right)+H_{n}^{d}\left(W_{\gamma, \mathbf{0}_{k}}\right),
\end{aligned}
$$

where $x_{d+1}=1-|x|$, and with $x_{0}:=1$ and $\left(\varepsilon_{1}, \ldots, \varepsilon_{d+1}\right)=\left(\mathbf{0}_{d-k+1},\left(\mathbf{0}_{k-j}, \mathbf{1}_{j}\right) \sigma\right)$,

$$
x_{j, k}(\sigma):=\prod_{i=1}^{d+1} x_{\delta_{i}} \quad \text { with } \quad \delta_{i}=i \text { if } \varepsilon_{i}=1 \text {, and } \delta_{i}=0 \text { if } \varepsilon_{i}=0 .
$$


(iii) if $k=d+1$, then $\mathcal{U}_{0}^{d}\left(W_{-1}\right)=\operatorname{span}\{1\}, \mathcal{U}_{1}^{d}\left(W_{-\mathbf{1}}\right)=\operatorname{span}\left\{x_{1}+c_{1}, \ldots, x_{d}+c_{d}\right\}$ for arbitrary real numbers $c_{1}, \ldots, c_{d}$, and for $n \geq 2$,

$$
\mathcal{U}_{n}^{d}\left(W_{-\mathbf{1}}\right)=x_{1} \cdots x_{d+1} \mathcal{V}_{n-d-1}^{d}\left(W_{\mathbf{1}}\right)+\sum_{j=2}^{d} \sum_{\sigma \in \mathcal{G}_{d+1}} x_{j, d+1}(\sigma) H_{n-j}^{d}\left(W_{\left(\mathbf{0}_{d+1-j}, \mathbf{1}_{j}\right) \sigma}\right)
$$

where, with $\left(\varepsilon_{1}, \ldots, \varepsilon_{d+1}\right)=\left(\mathbf{0}_{d+1-j}, \mathbf{1}_{j}\right) \sigma$,

$$
x_{j, d+1}(\sigma):=\prod_{i=1}^{d+1} x_{\delta_{i}} \quad \text { with } \quad \delta_{i}=i \text { if } \varepsilon_{i}=1, \text { and } \delta_{i}=0 \text { if } \varepsilon_{i}=0
$$

In the case of $k=d+1$, that is, all $\gamma_{j}=-1$, the differential equation becomes

$$
L_{-1} P=-n(n-1) P
$$

For $n=1$, any polynomial of the form $P_{i}(x)=x_{i}+c_{i}$ with $c_{i}$ being an arbitrary constant satisfies the equation $L_{-1} P=0$. The values of the constant will be fixed when we discuss the Sobolev orthogonality.

As an example, we give the decomposition for $k=3$ and $k<d$ explicitly:

$$
\begin{aligned}
\mathcal{U}_{n}^{d}\left(W_{\gamma,-1,-1,-1}\right)= & x_{d-1} x_{d} x_{d+1} \mathcal{V}_{n-3}^{d}\left(W_{\gamma, 1,1,1}\right)+x_{d-1} x_{d} H_{n-2}^{d}\left(W_{\gamma, 1,1,0}\right) \\
& +x_{d-1} x_{d+1} H_{n-2}^{d}\left(W_{\gamma, 1,0,1}\right)+x_{d} x_{d+1} H_{n-2}^{d}\left(W_{\gamma, 0,1,1}\right) \\
& +x_{d-1} H_{n-1}^{d}\left(W_{\gamma, 1,0,0}\right)+x_{d} H_{n-1}^{d}\left(W_{\gamma, 0,1,0}\right) \\
& +x_{d+1} H_{n-1}^{d}\left(W_{\gamma, 0,0,1}\right)+H_{n}^{d}\left(W_{\gamma, 0,0,0}\right) .
\end{aligned}
$$

Our next theorem shows that the elements in $\mathcal{U}_{n}^{d}\left(W_{\gamma,-\mathbf{1}_{k}}\right)$ are orthogonal polynomials with respect to an inner product of the Sobolev type. We need the following notations: for $1 \leq k \leq d-1$, define

$$
\mathbf{x}_{k}:=\left(x_{1}, \ldots, x_{k}\right), \quad\left|\mathbf{x}_{k}\right|=x_{1}+\ldots+x_{k}, \quad \gamma_{k}:=\left(\gamma_{1}, \ldots, \gamma_{k}\right)
$$

Recall that, for $I \subset \mathbb{Z}_{d+1}$ with $|I|=i \leq d$, the face $T_{I}^{d-i}$ of the simplex $T^{d}$ is defined by $T_{I}^{d-i}=\left\{x \in T^{d}: x_{\ell}=0, \ell \in I\right\}$. If $I=\left\{j_{1}, \ldots, j_{i}\right\}$, we define $\mathbf{x}_{I}:=\left(x_{j_{1}}, \ldots, x_{j_{i}}\right)$.

Definition 3.6. For $\gamma \in \mathbb{R}^{d}, \gamma_{d+1}=-1$ and $\lambda \geq 0$, define

$$
\langle f, g\rangle_{\gamma,-1}:=\sum_{i=1}^{d} \int_{T^{d}} x_{i} \frac{\partial f}{\partial x_{i}} \frac{\partial g}{\partial x_{i}} x^{\gamma} d x+\lambda \int_{T_{\{d+1\}}^{d-1}} f(x) g(x) \mathbf{x}_{d-1}^{\gamma_{d-1}}\left(1-\left|\mathbf{x}_{d-1}\right|\right)^{\gamma_{d}} d \mathbf{x}_{d-1}
$$


For $2 \leq k \leq d$, let $\mathbf{m}_{k}=\{d-k+2, \ldots, d\}$ and $\mathbf{m}_{k}^{+}=\{d-k+2, \ldots, d+1\}$. For $\gamma \in \mathbb{R}^{\overline{d+1-k}}$ and $\lambda, \lambda_{i}, \lambda_{I} \geq 0$, define

$$
\begin{aligned}
\langle f, g\rangle_{\gamma,-\mathbf{1}_{k}}:= & \int_{T^{d}} \frac{\partial^{k-1} f}{\partial x^{\mathbf{m}_{k}}} \frac{\partial^{k-1} g}{\partial x^{\mathbf{m}_{k}}}(1-|x|)^{\left|\mathbf{m}_{k}\right|-1} \mathbf{x}_{d+1-k}^{\gamma} d x \\
& +\sum_{\substack { i=1 \\
\begin{subarray}{c}{I \subset \mathbf{m}_{k} \\
|I|=i{ i = 1 \\
\begin{subarray} { c } { I \subset \mathbf { m } _ { k } \\
| I | = i } }\end{subarray}}^{k-2} \lambda_{I} \int_{T_{I^{c}}^{d-k+i+1}} \frac{\partial^{i} f}{\partial x^{I}} \frac{\partial^{i} g}{\partial x^{I}}(1-|x|)^{i-1} \mathbf{x}_{d+1-k}^{\gamma} d \mathbf{x}_{d-k+1} d \mathbf{x}_{I} \\
& +\sum_{i=1}^{d-k+1} \lambda_{i} \int_{T_{\mathbf{m}_{k}-k+1}^{d-k}} x_{i} \frac{\partial f}{\partial x_{i}} \frac{\partial g}{\partial x_{i}} \mathbf{x}_{d+1-k}^{\gamma} d \mathbf{x}_{d-k+1} \\
& +\lambda \int_{T_{\mathbf{m}_{k}^{+}}^{d-k}} f\left(\mathbf{x}_{d-k+1}^{+}, \mathbf{0}\right) g\left(\mathbf{x}_{d-k+1}, \mathbf{0}\right) \mathbf{x}_{d-k+1}^{\gamma} d \mathbf{x}_{d-k},
\end{aligned}
$$

where if $k=d$, we replace the last term by $\lambda f\left(e_{1}\right) g\left(e_{1}\right)$, with $\lambda \geq 0$. Finally, when all $\gamma_{i}=-1$, for $\lambda_{I} \geq 0$ and $\lambda_{i, 0} \geq 0$, define

$$
\begin{aligned}
\langle f, g\rangle_{-\mathbf{1}}:= & \int_{T^{d}} \frac{\partial^{d} f}{\partial x^{\mathbb{Z}_{d}}} \frac{\partial^{d} g}{\partial x^{\mathbb{Z}_{d}}}(1-|x|)^{d-1} d x \\
& +\sum_{i=1}^{d-1} \sum_{\substack{I \subset \mathbb{Z}_{d} \\
|I|=i}} \lambda_{I} \int_{T_{I^{c}}^{i}} \frac{\partial^{i} f}{\partial x^{I}} \frac{\partial^{i} g}{\partial x^{I}}(1-|x|)^{i-1} d \mathbf{x}_{I} \\
& +\lambda_{0,0} f\left(e_{0}\right) g\left(e_{0}\right)+\lambda_{1,0} f\left(e_{1}\right) g\left(e_{1}\right)+\ldots+\lambda_{d, 0} f\left(e_{d}\right) g\left(e_{d}\right) .
\end{aligned}
$$

In the following we shall write $\langle f, g\rangle_{\gamma,-\mathbf{1}_{k}}$ with $k=1,2, \ldots, d+1$ for all three cases. Whenever $\langle f, g\rangle_{\gamma,-\mathbf{1}_{k}}$ is an inner product, we can define a space of orthogonal polynomials. Our next result says that the space of orthogonal polynomials with respect to $\langle f, g\rangle_{\gamma,-\mathbf{1}_{k}}$ is exactly $\mathcal{U}_{n}^{d}\left(W_{\gamma,-\mathbf{1}_{k}}\right)$. First, however, we need to specify $\mathcal{U}_{1}^{d}\left(W_{-\mathbf{1}}\right)$ : We redefine

$$
\mathcal{U}_{1}^{d}\left(W_{-1}\right)=\operatorname{span}\left\{x_{1}+c_{1}, \ldots, x_{d}+c_{d}\right\} \quad \text { with } \quad c_{j}=-\frac{\lambda_{j, 0}}{\lambda_{0,0}+\lambda_{1,0}+\ldots+\lambda_{d, 0}} .
$$

Theorem 3.7. For $1 \leq k \leq d+1$, let $\gamma \in \mathbb{R}^{d+1-k}$ with $\gamma_{j}>-1,1 \leq j \leq d+1-k$.

(i) $\langle f, g\rangle_{\gamma,-\mathbf{1}_{k}}$ is an inner product if $\lambda, \lambda_{i}, \lambda_{I}>0$ for $1 \leq k \leq d$ and, in addition, at least one of $\lambda_{j, 0}$ is positive for $k=d+1$.

(ii) $\mathcal{U}_{n}^{d}\left(W_{\gamma,-\mathbf{1}_{k}}\right)$ is the space of orthogonal polynomials with respect to $\langle f, g\rangle_{\gamma,-\mathbf{1}_{k}}$.

(iii) In the case of $k=1$, the decomposition of (3.3) is orthogonal, that is,

$$
\mathcal{U}_{n}^{d}\left(W_{\gamma,-1}\right)=x_{d+1} \mathcal{V}_{n-1}^{d}\left(W_{\gamma, 1}\right) \bigoplus H_{n}^{d}\left(W_{\gamma, 0}\right)
$$

Remark 3.3. For $k \geq 2$, the direct sums in the decomposition of $\mathcal{U}_{n}^{d}\left(W_{\gamma,-\mathbf{1}_{k}}\right)$ is in general not orthogonal sums. In other word, the elements belong to different parts of the decomposition (3.4) may not be mutually orthogonal in general. On the other hand, the Gram-Schmid process is applicable if an orthonormal basis is desired.

It should be mentioned that, as stated in Remark 3.2, it is possible to add more terms or higher order derivatives in the definition of $\langle f, g\rangle_{\gamma,-\mathbf{1}_{k}}$ but we strike to keep the number minimal. 


\section{Lemmas And Proof of Theorems}

In the first subsection we prove several lemmas that will be needed for the proof of Theorem 3.5. The proofs of the main results are given in the subsequent sections.

4.1. Lemmas on Rodrigue basis. Our first two lemmas are analogues of (2.4) of one variable with one of the element in $\gamma$ being -1 .

Lemma 4.1. Let $\gamma \in \mathbb{R}^{d}$ and $\gamma_{d+1}=-1$. Then, for $n_{i} \geq 1,1 \leq i \leq d$,

$$
P_{\mathbf{n}}^{(\gamma,-1)}(x)=(1-|x|) \sum_{i=1}^{d} \frac{n_{i}\left(\gamma_{i}+n_{i}\right)}{|\mathbf{n}|} P_{\mathbf{n}-e_{i}}^{(\gamma, 1)}(x) .
$$

Proof. Applying the product rule

$$
\left(\frac{d}{d x}\right)^{n}[(1-x) g(x)]=(1-x) \frac{d^{n}}{d x^{n}} g(x)-n \frac{d^{n-1}}{d x^{n-1}} g(x) .
$$

multiple times within the Rodrigue formula, we obtain

$$
P_{\mathbf{n}}^{(\gamma,-1)}(x)=x^{-\gamma} \frac{\partial^{|\mathbf{n}|}}{\partial x^{\mathbf{n}}}\left[x^{\gamma+\mathbf{n}}(1-|x|)^{|\mathbf{n}|}\right]+x^{-\gamma} \sum_{i=1}^{d} n_{i} \frac{\partial^{|\mathbf{n}|-1}}{\partial x^{\mathbf{n}-e_{i}}}\left[x^{\gamma+\mathbf{n}}(1-|x|)^{|\mathbf{n}|-1}\right],
$$

in which the first term in the right hand can be written as

$$
\begin{aligned}
x^{-\gamma} \frac{\partial^{|\mathbf{n}|}}{\partial x^{\mathbf{n}}}\left[x^{\gamma+\mathbf{n}}(1-|x|)^{|\mathbf{n}|}\right] & =\frac{\left(n_{1}+\cdots+n_{d}\right)}{|\mathbf{n}|} x^{-\gamma} \frac{\partial^{|\mathbf{n}|}}{\partial x^{\mathbf{n}}}\left[x^{\gamma+\mathbf{n}}(1-|x|)^{|\mathbf{n}|}\right] \\
& =\frac{x^{-\gamma}}{|\mathbf{n}|} \sum_{i=1}^{d} n_{i} \frac{\partial^{|\mathbf{n}|-1}}{\partial x^{\mathbf{n}-e_{i}}}\left[\frac{\partial}{\partial x_{i}}\left(x^{\gamma+\mathbf{n}}(1-|x|)^{|\mathbf{n}|}\right)\right],
\end{aligned}
$$

so that, putting the two identities together, we conclude that

$$
\begin{aligned}
P_{\mathbf{n}}^{(\gamma,-1)}(x) & =\frac{x^{-\gamma}}{|\mathbf{n}|} \sum_{i=1}^{d} n_{i}\left(\gamma_{i}+n_{i}\right) \frac{\partial^{|\mathbf{n}|-1}}{\partial x^{\mathbf{n}-e_{i}}}\left[x^{\gamma+\mathbf{n}-e_{i}}(1-|x|)^{|\mathbf{n}|}\right] \\
& =(1-|x|) \sum_{i=1}^{d} \frac{n_{i}\left(\gamma_{i}+n_{i}\right)}{|\mathbf{n}|} P_{\mathbf{n}-e_{i}}^{(\gamma, 1)}(x)
\end{aligned}
$$

which completes the proof.

Lemma 4.2. Assume $\gamma_{i}=-1$ for some $i, 1 \leq i \leq d$. Then for $n_{i} \geq 1$,

$$
P_{\mathbf{n}}^{\left(\gamma, \gamma_{d+1}\right)}(x)=-\left(|\mathbf{n}|+\gamma_{d+1}\right) x_{i} P_{\mathbf{n}-e_{i}}^{\left(\gamma+2 e_{i}, \gamma_{d+1}\right)}(x) .
$$

Proof. Without loss of generality, we can assume $i=d$. Setting $\gamma:=\left(\gamma_{d-1},-1\right)$ and $x=\left(\mathbf{x}_{d-1}, x_{d}\right)$, and applying the product rule

$$
\left(\frac{d}{d x}\right)^{n}[x g(x)]=x \frac{d^{n}}{d x^{n}} g(x)+n \frac{d^{n-1}}{d x^{n-1}} g(x),
$$

multiple time with the Rodrigue formula, we obtain

$$
\begin{aligned}
P_{\mathbf{n}}^{\left(\gamma, \gamma_{d+1}\right)}(x)= & \mathbf{x}_{d-1}^{-\gamma_{d-1}}(1-|x|)^{-\gamma_{d+1}} \frac{\partial^{|\mathbf{n}|}}{\partial x^{\mathbf{n}}}\left[x^{\gamma+\mathbf{n}+e_{d}}(1-|x|)^{|\mathbf{n}|+\gamma_{d+1}}\right] \\
& -n_{d} \mathbf{x}_{d-1}^{-\gamma_{d-1}}(1-|x|)^{-\gamma_{d+1}} \frac{\partial^{|\mathbf{n}|-1}}{\partial x^{\mathbf{n}-e_{d}}}\left[x^{\gamma+\mathbf{n}}(1-|x|)^{|\mathbf{n}|+\gamma_{d+1}}\right] .
\end{aligned}
$$


Splitting the partial derivatives in the first term as $\frac{\partial^{|\mathbf{n}|}}{\partial x^{\mathbf{n}}}=\frac{\partial^{|\mathbf{n}|-1}}{\partial x^{\mathbf{n}-e_{d}}} \frac{\partial}{\partial x_{d}}$, a quick computations establishes the stated identity.

By repeatedly applying the above lemmas, we can deduce a relation when more than one $\gamma_{i}$ equal to -1 . We state the results in the following corollaries.

Corollary 4.3. Let $\gamma \in \mathbb{R}^{d+1-k}$. For $n_{d-k+2} \geq 1, \ldots, n_{d} \geq 1$,

$$
\begin{aligned}
P_{\mathbf{n}}^{\left(\gamma,-\mathbf{1}_{k-1}, \gamma_{d+1}\right)}(x)= & (-1)^{k-1} x_{d+2-k} \cdots x_{d} \\
& \times \prod_{j=1}^{k-1}\left(|\mathbf{n}|+\gamma_{d+1}-j+1\right) P_{\mathbf{n}-\left(\mathbf{0}, \mathbf{1}_{k-1}\right)}^{\left(\gamma, \boldsymbol{1}_{k+1}\right)}(x) .
\end{aligned}
$$

In particular, it follows that

$$
x_{d+2-k} \cdots x_{d} \mathcal{V}_{n-k+1}^{d}\left(W_{\gamma, \mathbf{1}_{k-1}, \gamma_{d+1}}\right) \subset \mathcal{V}_{n}^{d}\left(W_{\gamma,-\mathbf{1}_{k-1}, \gamma_{d+1}}\right) .
$$

Combining Lemmas 4.1 and 4.2 , we can also deduce the following lemma:

Corollary 4.4. Let $\gamma \in \mathbb{R}^{d+1-k}$. For $n_{d-k+2} \geq 1, \ldots, n_{d} \geq 1$,

$$
P_{\mathbf{n}}^{\left(\gamma,-\mathbf{1}_{k}\right)}(x)=x_{d+2-k} \cdots x_{d}(1-|x|) \sum_{i=1}^{d} \lambda_{n_{i}, \gamma_{i}} P_{\mathbf{n}-e_{i}-\left(\mathbf{0}, \mathbf{1}_{k-1}\right)}^{\left(\gamma, \mathbf{1}_{k}\right)}(x),
$$

where, for $1 \leq i \leq d$,

$$
\lambda_{n_{i}, \gamma_{i}}=(-1)^{k-1} \frac{n_{i}\left(n_{i}+\gamma_{i}\right)}{|\mathbf{n}|} \prod_{j=0}^{k-2}(|\mathbf{n}|-j) .
$$

For later use, we need to reverse the expression in (4.1), which is the content of the following lemma.

Lemma 4.5. Let $\mathbf{j}=\left(j_{2}, \ldots, j_{d}\right)$. Then there exist constant $\mu_{\mathbf{j}}$ such that

$$
x_{d+2-k} \cdots x_{d}(1-|x|) P_{\mathbf{n}}^{\left(\gamma, \mathbf{1}_{k}\right)}(x)=\sum_{j_{i} \leq n_{i}} \mu_{\mathbf{j}} P_{(|\mathbf{n}|-|\mathbf{j}|+1, \mathbf{j})+\left(\mathbf{0}, \mathbf{1}_{k-1}\right)}^{\left(\gamma, \mathbf{1}_{k}\right)}(x),
$$

where the sum is over $j_{i}$ for $i=2,3, \ldots, d$. In particular, for $n \geq k$,

$$
x_{d+2-k} \cdots x_{d}(1-|x|) \mathcal{V}_{n-k}^{d}\left(W_{\gamma, \mathbf{1}_{k}}\right) \subset \mathcal{V}_{n}^{d}\left(W_{\gamma,-\mathbf{1}_{k}}\right) .
$$

Proof. To simplify the notation in the proof, let

$$
X_{k}:=x_{d-k+2} \cdots x_{d}(1-|x|), \quad \mathbf{n}_{k}:=\left(n_{1}, \ldots, n_{k}\right) \in \mathbb{N}_{0}^{k} .
$$

From (4.1) it follows that

$$
P_{\left(n, \mathbf{0}_{d-k}, \mathbf{1}\right)}^{\left(\gamma,-\mathbf{1}_{k}\right)}=X_{k} \lambda_{n, \gamma_{1}} P_{(n-1, \mathbf{0})}^{\left(\gamma, \mathbf{1}_{k}\right)}(x),
$$

which proves (4.2) for $\mathbf{n}=(n, 0, \ldots, 0)$. Notice that the subindex $\mathbf{n}$ of $P_{\mathbf{n}}^{\gamma}$ is always in $\mathbb{N}_{0}^{d}$, so that we have suppressed the subindex of $\mathbf{1}=\mathbf{1}_{k-1}$ for the polynomial in the left hand side and the subindex of $\mathbf{0}=\mathbf{0}_{d-1}$ for the polynomial in the right hand side. We shall keep this convention below. Again by (4.1), we have

$$
P_{\left(n_{1}, n_{2}, \mathbf{0}_{d-(k+1)}, \mathbf{1}\right)}^{\left(\gamma,-\mathbf{1}_{k}\right)}=X_{k}\left[\lambda_{n_{1}, \gamma_{1}} P_{\left(n_{1}-1, n_{2}, \mathbf{0}\right)}^{\left(\gamma, \mathbf{1}_{k}\right)}(x)+\lambda_{n_{2}, \gamma_{2}} P_{\left(n_{1}, n_{2}-1, \mathbf{0}\right)}^{\left(\gamma, \mathbf{1}_{k}\right)}(x)\right],
$$

which shows by induction on $n_{2}$ that

$$
X_{k} P_{\left(n_{1}, n_{2}, \mathbf{0}\right)}^{\left(\gamma, \mathbf{1}_{k}\right)}(x)=\sum_{j=0}^{n_{2}} \mu_{j} P_{\left(n_{1}+n_{2}-j+1, j, \mathbf{0}_{d-(k+1)}, \mathbf{1}\right)}^{\left(\gamma,-\mathbf{1}_{k}\right)}(x),
$$


where the $\mu_{j}$ can be determined inductively, which proves (4.2) for $\mathbf{n}=\left(n_{1}, n_{2}, \ldots, 0\right)$. Furthermore, the above proof shows, in fact, that

$$
X_{k} P_{\left(\left(n_{1}, n_{2}, \mathbf{0}_{d-(k+1)}\right) \sigma, \mathbf{0}\right)}^{\left(\gamma, \mathbf{1}_{k}\right)}(x)=\sum_{j=0}^{n_{2}} \mu_{j} P_{\left(\left(n_{1}+n_{2}-j+1, j, \mathbf{0}_{d-(k+1)}\right) \sigma, \mathbf{1}\right)}^{\left(\gamma,-\mathbf{1}_{k}\right)}(x)
$$

for every $\sigma \in \mathcal{G}_{d-k+1}$, the permutation group of $(d-k+1)$ elements. In the next step, we deduce from (4.1) that

$$
\begin{aligned}
P_{\left(n_{1}, n_{2}, n_{3}, \mathbf{0}_{d-(k+2)}, \mathbf{1}\right)}^{\left(\gamma,-\mathbf{1}_{k}\right)}(x)= & X_{k}\left[\lambda_{n_{1}, \gamma_{1}} P_{\left(n_{1}-1, n_{2}, n_{3}, \mathbf{0}\right)}^{\left(\gamma, \mathbf{1}_{k}\right)}(x)\right. \\
& \left.+\lambda_{n_{2}, \gamma_{2}} P_{\left(n_{1}, n_{2}-1, n_{3}, \mathbf{0}\right)}^{\left(\gamma, \mathbf{1}_{k}\right)}(x)+\lambda_{n_{3}, \gamma_{3}} P_{\left(n_{1}, n_{2}, n_{3}-1, \mathbf{0}\right)}^{\left(\gamma, \mathbf{1}_{k}\right)}(x)\right] .
\end{aligned}
$$

For $n_{3}=1$, we can use induction on $n_{2}$ to deduce, from $X_{k} P_{\left(\left(n_{1}, n_{2}, \mathbf{0}_{d-(k+1)}\right) \sigma, \mathbf{0}\right)}^{\left(\gamma, \mathbf{1}_{k}\right)}(x)$, the desired (4.2) for $X_{k} P_{\left(n_{1}, n_{2}, 1, \mathbf{0}\right)}^{\left(\gamma, \mathbf{1}_{1}\right)}(x)$ and, hence, for $X_{k} P_{\left(\left(n_{1}, n_{2}, 1, \mathbf{0}_{d-(k+2)}\right) \sigma, \mathbf{0}\right)}^{\left(\gamma, \mathbf{1}_{1}\right)}(x)$. Then, by induction on $n_{3}$, we can deduce the desired (4.2) for $\mathbf{n}=\left(n_{1}, n_{2}, n_{3}, \mathbf{0}\right)$. It is now clear how to proceed, by induction if necessary, to conclude that (4.2) holds for $\mathbf{n}=\left(\mathbf{n}_{d-k}, \mathbf{0}\right)$.

We can go beyond $d-k$ by using (4.1) and the fact that $\lambda_{m, \tau}=0$ if $m=1$ and $\tau=-1$. Indeed, (4.1) implies that

$$
P_{\left(\mathbf{n}_{d-k+1}, \mathbf{1}\right)}^{\left(\boldsymbol{\gamma},-\mathbf{1}_{k}\right)}(x)=X_{k} \sum_{i=1}^{d-k+1} \lambda_{n_{i}, \gamma_{i}} P_{\left(\mathbf{n}_{d-k+1}, \mathbf{0}\right)-e_{i}}^{\left(\boldsymbol{\gamma}, \mathbf{1}_{k}\right)}(x),
$$

which allows us to use the induction on $n_{d-k+1}$ to prove (4.2) for $\mathbf{n}=\left(\mathbf{n}_{d-k+1}, \mathbf{0}\right)$. Furthermore, the process can obviously be continued, by induction if necessary, until we reach $\mathbf{n}=\left(n_{1}, \ldots, n_{d}\right)$. This completes the proof.

4.2. Proof of Theorem 3.5. By (3.4) in the Theorem 3.5, the space $\mathcal{U}_{n}^{d}\left(W_{\gamma,-\mathbf{1}_{k}}\right)$ consists of three main terms. Since, by Lemma 4.5, the elements in the space $x_{d+2-k} \cdots x_{d+1} \mathcal{V}_{n-k}^{d}\left(W_{\gamma, \mathbf{1}_{k}}\right)$ are orthogonal polynomials of degree $n$ in $\mathcal{V}_{n}^{d}\left(W_{\gamma,-\mathbf{1}_{k}}\right)$, by Remark 2.1, they satisfy the equation

$$
L_{\gamma,-\mathbf{1}_{k}} u=-n(n+|\gamma|+d-k) u,
$$

which takes care of the first term. The second term of the decomposition in (3.4) is a large sum. Since the permutation is acting on $\left(\mathbf{0}_{k-j}, \mathbf{1}_{j}\right)$ and the differential operator $L_{\gamma, \mathbf{0}_{k-j}, \mathbf{1}_{j}}$ is clearly invariant under such permutation, we only need to consider, for $1 \leq j \leq k-1$, the cases of $x_{j, k} H_{n-j}^{d}\left(W_{\gamma, \mathbf{0}_{k-j}, \mathbf{1}_{j}}\right)$ with $x_{j, k}=x_{d+2-j} \cdots x_{d+1}$ and $x_{j, k} H_{n-j}^{d}\left(W_{\gamma, \mathbf{0}_{k-j-1}, \mathbf{1}_{j}, 0}\right)$ with $x_{j, k}=x_{d+1-j} \cdots x_{d}$. We consider the case of $x_{d+2-j} \cdots x_{d+1} H_{n-j}^{d}\left(W_{\gamma, \mathbf{0}_{k-j}, \mathbf{1}_{j}}\right)$ first. Recall that

$H_{n-j}^{d}\left(W_{\gamma, \mathbf{0}_{k-j}, \mathbf{1}_{j}}\right)=H_{n-j, S_{k, j}}^{d}\left(W_{\gamma, \mathbf{0}_{k-j}, \mathbf{1}_{j}}\right) \quad$ with $\quad S_{k, j}=\{d-k+2, \ldots, d-j+1\}$.

From Lemma 2.11, since the parameters in $\left\{\gamma_{i}: i \in S_{k, j}\right\}$ do not appear in the basis of $H_{n-j}^{d}\left(W_{\gamma, \mathbf{0}_{k-j}, \mathbf{1}_{j}}\right)$, the values of these parameters can be arbitrary. As a result, we can write

$$
H_{n-j}^{d}\left(W_{\gamma, \mathbf{0}_{k-j}, \mathbf{1}_{j}}\right)=H_{n-j}^{d}\left(W_{\gamma,-\mathbf{1}_{k-j}, \mathbf{1}_{j}}\right) .
$$

On the other hand, from Lemma 4.5. for $\gamma \in \mathbb{R}^{d-k+1}$, we have

$$
x_{d+2-j} \cdots x_{d}(1-|x|) \mathcal{V}_{n-j}^{d}\left(W_{\gamma,-\mathbf{1}_{k-j}, \mathbf{1}_{j}}\right) \subset \mathcal{V}_{n}^{d}\left(W_{\gamma,-\mathbf{1}_{k}}\right) .
$$


Since, by Lemma 2.10, $H_{n-j}^{d}\left(W_{\gamma,-\mathbf{1}_{k-j}, \mathbf{1}_{j}}\right) \subset \mathcal{V}_{n-j}^{d}\left(W_{\gamma,-\mathbf{1}_{k-j}, \mathbf{1}_{j}}\right)$, together with (4.4) it follows that

$$
x_{d+2-j} \cdots x_{d}(1-|x|) H_{n-j}^{d}\left(W_{\gamma, \mathbf{0}_{k-j}, \mathbf{1}_{j}}\right) \subset \mathcal{V}_{n}^{d}\left(W_{\gamma,-\mathbf{1}_{k}}\right),
$$

so that the elements of $x_{d+2-j} \cdots x_{d}(1-|x|) H_{n-j}^{d}\left(W_{\gamma, \mathbf{o}_{k-j}, \mathbf{1}_{j}}\right)$ satisfy the equation (4.3). Secondly, we consider the case of $x_{d+1-j} \cdots x_{d} H_{n-j}^{d}\left(W_{\gamma, \mathbf{o}_{k-j-1}, \mathbf{1}_{j}, 0}\right)$. Similar to the first case, as a result of Corollary 4.3 and Lemma 2.11. we can write

$$
x_{d+1-j} \cdots x_{d} H_{n-j}^{d}\left(W_{\gamma, \mathbf{0}_{k-j-1}, \mathbf{1}_{j}, 0}\right) \subset \mathcal{V}_{n}^{d}\left(W_{\gamma,-\mathbf{1}_{k}}\right),
$$

so that these polynomials satisfy the equation (4.3) for $j=1,2, \ldots, k-1$. Finally, the elements of the third term $H_{n}^{d}\left(W_{\gamma, \mathbf{o}_{k}}\right)$ in the decomposition of $\mathcal{U}_{n}^{d}\left(W_{\gamma},-\mathbf{1}_{k}\right)$ satisfy the equation (4.3) according to Lemma 2.11.

The same proof applies to the case of $k=d+1$ for $n>1$, whereas the case $\mathcal{U}_{1}^{d}\left(W_{-1}\right)$ has been explained right below the statement of Theorem 3.5 .

It is remain to prove that the $\mathcal{U}_{n}^{d}\left(W_{\gamma,-\mathbf{1}_{k}}\right)$ has full dimension. From Lemma 2.10, the restriction of $H_{n}^{d}\left(W_{\gamma,\left(\mathbf{0}_{k-j}, \mathbf{1}_{j}\right) \sigma}\right)$ on the face $T_{S_{k, j}(\sigma)}^{d-(k-j)}$ is isomorphic to $\mathcal{V}_{n}^{d-(k-j)}\left(W_{\gamma, \mathbf{1}_{j}}\right)$, so that

$$
\operatorname{dim} H_{n}^{d}\left(W_{\gamma,\left(\mathbf{o}_{k-j}, \mathbf{1}_{j}\right) \sigma}\right)=\left(\begin{array}{c}
n+d-(k-j+1) \\
n
\end{array}\right),
$$

which implies, together with $\operatorname{dim} \mathcal{V}_{n}^{d}\left(W_{\gamma}\right)=\left(\begin{array}{c}n+d-1 \\ n\end{array}\right)$, that

$$
\begin{aligned}
\operatorname{dim} \mathcal{U}_{n}^{d}\left(W_{\gamma,-\mathbf{1}_{k}}\right)= & \operatorname{dim} \mathcal{V}_{n-k}^{d}\left(W_{\gamma, \mathbf{1}_{k}}\right) \\
& +\sum_{j=1}^{k-1}\left(\begin{array}{c}
k \\
j
\end{array}\right) \operatorname{dim} H_{n-j}^{d}\left(W_{\gamma,\left(\mathbf{0}_{k-j}, \mathbf{1}_{j}\right) \sigma}\right)+\operatorname{dim} H_{n}^{d}\left(W_{\gamma, \mathbf{o}_{k}}\right) \\
= & \sum_{j=0}^{k}\left(\begin{array}{c}
k \\
j
\end{array}\right)\left(\begin{array}{c}
n+d-k-1 \\
n-j
\end{array}\right)=\left(\begin{array}{c}
n+d-1 \\
n
\end{array}\right),
\end{aligned}
$$

where the last step uses the well-known combinatorial identity.

4.3. Proof of (i) in Theorem 3.7. To show that each bilinear form is an inner product, we only need to show that it preserves positivity. Since all coefficients are assumed to be non-negative, we clearly have $\langle f, f\rangle_{\gamma,-\mathbf{1}_{k}} \geq 0$ and it remains to show that $\langle f, f\rangle_{\gamma,-\mathbf{1}_{k}}=0$ implies that $f \equiv 0$.

Assume that $k=1$ and $\langle f, f\rangle_{\gamma,-1}=0$. Then, by the definition, $\frac{\partial f}{\partial x_{i}}=0$ for $1 \leq i \leq d$, which implies that $f$ is a constant. Furthermore, the last term of $\langle f, f\rangle_{\gamma,-1}$ shows then $f \equiv 0$. Thus, $\langle f, f\rangle_{\gamma,-1}$ is an inner product.

Now assume that $2 \leq k \leq d$ and $\langle f, f\rangle_{\gamma,-\mathbf{1}_{k}}=0$. Then each term of in the right hand side of $\langle f, f\rangle_{\gamma,-\mathbf{1}_{k}}$ is zero. There are four main terms. The first term shows $\frac{\partial^{k-1} f}{\partial x^{\mathbf{m}_{k}}}=0$, which implies that $f$ has the form

$$
f(x)=\sum_{\substack{J \subset \mathbf{m}_{k} \\|J|=k-2}} f_{J}\left(x_{J}\right), \quad x_{J} \in T_{J^{c}}^{d-1} .
$$


The second term with $|J|=k-2$ shows $\frac{\partial^{k-2} f}{\partial x^{J}}=0$, which implies that $f$ has the form

$$
f(x)=\sum_{\substack{J \subset \mathbf{m}_{k} \\|J|=k-3}} f_{J}\left(x_{J}\right), \quad x_{J} \in T_{J^{c}}^{d-2},
$$

we can continue this process with $|J|=j$ for $j=k-3, \ldots, 1$ and deduce from the second term of $\langle f, f\rangle_{\gamma,-\mathbf{1}_{k}}$ that $f$ has the form $f(x)=f_{1}\left(x_{1}, \ldots, x_{d-k+1}\right)$. Now, the third term of $\langle f, f\rangle_{\gamma,-\mathbf{1}_{k}}$ shows that $\frac{\partial f}{\partial x_{j}}=0$ for $j=1, \ldots, d-k+1$, from which it follows then that $f$ is a constant. Finally, the fourth term of $\langle f, f\rangle_{\gamma,-\mathbf{1}_{k}}$ shows that $f(x)=0$ if $k<d$ or if $k=d$ and $\lambda>0$. Thus $\langle f, g\rangle_{\gamma,-\mathbf{1}_{k}}$ is an inner product. The same proof clearly applies to $\langle f, g\rangle_{-1}$.

4.4. Proof of (ii) in Theorem 3.7. We need to show that, for each $k$, the polynomial spaces of $\mathcal{U}_{n}^{d}\left(W_{\gamma,-\mathbf{1}_{k}}\right)$ given in Theorem 3.5 are the spaces of orthogonal polynomials with respect to the inner product $\langle\cdot, \cdot\rangle_{\gamma,-\mathbf{1}_{k}}$. The proof is involved and will be divided into several cases. Throughout the proof, we let $g$ be a generic element in $\Pi_{n-1}^{d}$.

Case 1. $k=1$. The inner product $\langle f, g\rangle_{\gamma,-1}$ contains two terms,

$$
\langle f, g\rangle_{\gamma,-1}=[f, g]_{1}+[f, g]_{2},
$$

where

$$
\begin{aligned}
{[f, g]_{1} } & :=\sum_{i=1}^{d} \int_{T^{d}} x_{i} \frac{\partial f}{\partial x_{i}} \frac{\partial g}{\partial x_{i}} x^{\gamma} d x \\
{[f, g]_{2} } & :=\lambda \int_{T_{\{d+1\}}^{d-1}} f(x) g(x) \mathbf{x}_{d-1}^{\gamma_{d-1}}\left(1-\left|\mathbf{x}_{d-1}\right|\right)^{\gamma_{d}} d \mathbf{x}_{d-1} .
\end{aligned}
$$

By Theorem 3.5, the space of $\mathcal{U}_{n}^{d}\left(W_{\gamma,-1}\right)$ with $\gamma_{i}>-1,1 \leq i \leq d$, is decomposed into two terms as shown in (3.4). The proof is divided into two cases accordingly.

Case 1.1. Let $f(x)=(1-|x|) P_{\mathbf{n}}^{(\gamma, 1)}(x)$, where $P_{\mathbf{n}}^{(\gamma, 1)}(x) \in \mathcal{V}_{n-1}^{d}\left(W_{\gamma, 1}\right)$ and $\mathbf{n} \in \mathbb{N}_{0}^{d}$ and $|\mathbf{n}|=n-1$. Then the restriction of $f$ on $|x|=1$ is zero so that $[f, g]_{2}=0$ for all $g$. Furthermore, an integration by parts gives

$$
[f, g]_{1}=-\sum_{i=1}^{d} \int_{T^{d}} x^{\gamma}(1-|x|) P_{\mathbf{n}}^{(\gamma, 1)}(x)\left\{x_{i} \frac{\partial^{2} g}{\partial x_{i}{ }^{2}}+\left(\gamma_{i}+1\right) \frac{\partial g}{\partial x_{i}}\right\} d x .
$$

Since the term in the curly bracket is a polynomial of degree at most $n-2$, the orthogonality of $P_{\mathbf{n}}^{(\gamma, 1)}(x) \in \mathcal{V}_{n-1}^{d}\left(W_{\gamma, 1}\right)$ implies immediately that $[f, g]_{1}=0$, so that $\langle f, g\rangle_{\gamma, 1}=0$ in this case.

Case 1.2. Let $f \in H_{n}^{d}\left(W_{\gamma, 0}\right)$. By Lemma 2.10 the restriction of $H_{n}^{d}\left(W_{\gamma, 0}\right)$ on the plane $|x|=1$ is exactly $\mathcal{V}_{n}^{d-1}\left(W_{\gamma}\right)$. It follows immediately that $[f, g]_{2}=0$ for all $g \in \Pi_{n-1}^{d}$. Furthermore, an integration by parts gives

$$
[f, g]_{1}=-\sum_{i=1}^{d} \int_{T^{d}} x^{\gamma} f(x)\left\{x_{i} \frac{\partial^{2} g}{\partial x_{i}{ }^{2}}+\left(\gamma_{i}+1\right) \frac{\partial g}{\partial x_{i}}\right\} d x .
$$

Since $f \in \mathcal{V}_{n}^{d}\left(W_{\gamma, 0}\right)$ by Lemma2.10, it follows that $[f, g]_{1}=0$, so that $\langle f, g\rangle_{\gamma,-1}=0$. 
By the decomposition of $\mathcal{U}_{n}^{d}\left(W_{\gamma,-1}\right)$, the two cases imply that $\langle f, g\rangle_{\gamma,-1}=0$ for all $f \in \mathcal{U}_{n}^{d}\left(W_{\gamma,-1}\right)$ and $g \in \Pi_{n-1}^{d}$, which completes the proof in this case.

Case 2. $2 \leq k \leq d$. The inner product $\langle f, g\rangle_{\gamma,-\mathbf{1}_{k}}$ contains four terms,

$$
\langle f, g\rangle_{\gamma,-1}=[f, g]_{1}+[f, g]_{2}+[f, g]_{3}+[f, g]_{4}
$$

where

$$
\begin{aligned}
{[f, g]_{1} } & :=\int_{T^{d}} \frac{\partial^{k-1} f}{\partial x^{\mathbf{m}_{k}}} \frac{\partial^{k-1} g}{\partial x^{\mathbf{m}_{k}}}(1-|x|)^{\left|\mathbf{m}_{k}\right|-1} \mathbf{x}_{d+1-k}^{\gamma} d x \\
{[f, g]_{2} } & :=\sum_{i=1}^{k-2} \sum_{\substack{I \subset \mathbf{m}_{k} \\
|I|=i}} \lambda_{I} \int_{T_{I^{c}}^{d-k+i+1}} \frac{\partial^{i} f}{\partial x^{I}} \frac{\partial^{i} g}{\partial x^{I}}(1-|x|)^{i-1} \mathbf{x}_{d+1-k}^{\gamma} d \mathbf{x}_{d-k+1} d \mathbf{x}_{I}, \\
{[f, g]_{3}:=} & \sum_{i=1}^{d-k+1} \lambda_{i} \int_{T_{\mathbf{m}_{k}}^{d-k+1}} x_{i} \frac{\partial f}{\partial x_{i}} \frac{\partial g}{\partial x_{i}} \mathbf{x}_{d+1-k}^{\gamma} d \mathbf{x}_{d-k+1}, \\
{[f, g]_{4}:=} & \lambda \int_{T_{\mathbf{m}_{k}^{+}}^{d-k}} f\left(\mathbf{x}_{d-k+1}, \mathbf{0}\right) g\left(\mathbf{x}_{d-k+1}, \mathbf{0}\right) \mathbf{x}_{d-k+1}^{\gamma} d \mathbf{x}_{d-k},
\end{aligned}
$$

where $\mathbf{m}_{k}=\{d-k+2, \ldots, d\}$ and $\mathbf{m}_{k}^{+}=\{d-k+2, \ldots, d+1\}$. By Theorem 3.5] the space $\mathcal{U}_{n}^{d}\left(W_{\gamma,-\mathbf{1}_{k}}\right), \gamma_{i}>-1$ for $1 \leq i \leq d+1-k$, is decomposed into three pieces as in (3.4), where the second term is a large sum. The proof is divided into several cases according to this decomposition.

Case 2.1. $f$ is an element of $x_{d-k+2} \cdots x_{d+1} \mathcal{V}_{n-k}^{d}\left(W_{\gamma, \mathbf{1}_{k}}\right)$, the first term of (3.4). Let $f(x)=x_{d-k+2} \cdots x_{d} x_{d+1} P_{\mathbf{n}}^{\left(\gamma, \mathbf{1}_{k}\right)}(x)$, where $x_{d+1}=(1-|x|)$ and $P_{\mathbf{n}}^{\left(\gamma, \mathbf{1}_{k}\right)}(x) \in$ $\mathcal{V}_{n-k}^{d}\left(W_{\gamma, \mathbf{1}_{k}}\right)$ with $\mathbf{n} \in \mathbb{N}_{0}^{d}$ and $|\mathbf{n}|=n-k$.

For $I \subset \mathbf{m}_{k}$ with $|I|=i$, the variables on indices in $I^{c}=\mathbf{m}_{k} \backslash I$ appear as product factors in $\frac{\partial^{i} f}{\partial x^{I}}$ so that the restriction of $\frac{\partial^{i} f}{\partial x^{I}}$ on the face $T_{I^{c}}^{d-k+i+1}$ is zero; consequently, $[f, g]_{2}=0$. For $1 \leq i \leq d-k+1$, the variables $x_{d-k+2}, \ldots, x_{d}$ appear as product factors in $\frac{\partial f}{\partial x_{i}}$, so that $\frac{\partial f}{\partial x_{i}}$ vanishes on the face $T_{\mathbf{m}_{k}}^{d-k+1}$; consequently $[f, g]_{3}=0$. Furthermore, since $f$ vanishes whenever one of $x_{d-k+2}, \ldots, x_{d}, x_{d+1}$ is zero, it follows that $[f, g]_{4}=0$. Thus, it remains to consider $[f, g]_{1}$. From the Rodrigue formula (2.9) of $P_{\mathbf{n}}^{\left(\gamma, \mathbf{1}_{k}\right)}(x)$, it follows that

$$
f(x)=\mathbf{x}_{d-k+1}^{-\gamma} \frac{\partial^{|\mathbf{n}|}}{\partial x^{\mathbf{n}}}\left\{x^{\left(\gamma, \mathbf{1}_{k-1}\right)+\mathbf{n}}(1-|x|)^{|\mathbf{n}|+1}\right\} .
$$

Taking derivative of $f$ with respect to variables whose index is in $\mathbf{m}_{k}$, we obtain

$$
\begin{aligned}
\frac{\partial^{k-1} f}{\partial x^{\mathbf{m}_{k}}}=\frac{\partial^{k-1} f}{\partial x_{d-k+2} \cdots x_{d}} & =\mathbf{x}_{d-k+1}^{-\gamma} \frac{\partial^{n-1}}{\partial x^{\mathbf{n}+\left(\mathbf{0}, \mathbf{1}_{k-1}\right)}}\left\{x^{\left(\gamma, \mathbf{1}_{k-1}\right)+\mathbf{n}}(1-|x|)^{|\mathbf{n}|+1}\right\} \\
& =(1-|x|)^{-(k-2)} P_{\mathbf{n}+\left(\mathbf{0}, \mathbf{1}_{k-1}\right)}^{(x),-(k-2))}(x),
\end{aligned}
$$

from which we obtain immediately that

$$
\begin{aligned}
{[f, g]_{1} } & =\int_{T^{d}} \frac{\partial^{k-1} f}{\partial x^{\mathbf{m}_{k}}} \frac{\partial^{k-1} g}{\partial x^{\mathbf{m}_{k}}}(1-|x|)^{k-2} \mathbf{x}_{d+1-k}^{\gamma} d x \\
& =\int_{T^{d}} \mathbf{x}_{d+1-k}^{\gamma} P_{\mathbf{n}+\left(\mathbf{0}, \mathbf{1}_{k-1}\right)}^{\left(\gamma, \mathbf{0}_{k-1},-(k-2)\right)}(x) \frac{\partial^{k-1} g}{\partial x^{\mathbf{m}_{k}}} d x .
\end{aligned}
$$


Since $P_{\mathbf{n}+\left(\mathbf{0}, \mathbf{1}_{k-1}\right)}^{\left(\gamma, \mathbf{0}_{k-1},-(k-2)\right)}(x)$ is a polynomial of degree $(n-1)$ and

$$
n-1>(k-2)+n-k=(k-2)+\operatorname{deg} \frac{\partial^{k-1} g}{\partial x^{\mathbf{m}_{k}}}
$$

for $g \in \Pi_{n-1}^{d}$, it follows from Lemma 2.3 that $[f, g]_{1}=0$. This completes the proof in this case.

Case 2.2. $f$ is an element of $x_{j, k}(\sigma) H_{n-j}^{d}\left(W_{\gamma,\left(\mathbf{0}_{k-j}, \mathbf{1}_{j}\right) \sigma}\right)$, where $1 \leq j \leq k-1$ and $\sigma \in \mathcal{G}_{k}$, and $x_{j, k}$ contains the variable $x_{d+1}$.

First of all, since the inner product $\langle f, g\rangle_{\gamma, \mathbf{1}_{k}}$ involves symmetric derivatives with respect to variables $\left\{x_{d-k+2}, \ldots, x_{d}\right\}$, it is sufficient to consider the case that $\sigma=i d$. Let $j$ be fixed, $1 \leq j \leq k-1$. Since $x_{j, k}$ contains the variable $x_{d+1}$, we can assume then

$$
x_{j, k}=x_{d+2-j} \cdots x_{d} x_{d+1} \quad \text { and } \quad f(x)=x_{d+2-j} \cdots x_{d} x_{d+1} Q_{n-j}(x)
$$

where $Q_{n-j} \in H_{n-j}^{d}\left(W_{\gamma, \mathbf{0}_{k-j}, \mathbf{1}_{j}}\right)$. From Definition 2.9 with $S_{j}$ being the set $S_{k, j}=$ $\{d-k+2, \ldots, d+1-j\}$, we have then

$H_{n-j}^{d}\left(W_{\gamma, \mathbf{0}_{k-j}, \mathbf{1}_{j}}\right)=\left\{P_{\mathbf{n}}^{\left(\gamma, \mathbf{0}_{k-j}, \mathbf{1}_{j}\right)}(x): \mathbf{n} \in \mathbb{N}_{0}^{d},|\mathbf{n}|=n-j \quad\right.$ with $\left.n_{i}=0, i \in S_{k, j}\right\}$.

To keep the notation compact, we introduce the following notations in the proof. Recall that for $x \in \mathbb{R}^{d}, \mathbf{n} \in \mathbb{N}_{0}^{d}$ and $1 \leq i \leq d, \mathbf{x}_{i}=\left(x_{1}, \ldots, x_{i}\right)$ and $\mathbf{n}_{i}=$ $\left(n_{1}, \ldots, n_{i}\right)$. For $1 \leq i \leq d$, we set

$$
\overleftarrow{\mathbf{x}}_{i}:=\left(x_{i}, \ldots, x_{d}\right) \quad \text { and } \quad \overleftarrow{\mathbf{n}}_{i}:=\left(n_{i}, \ldots, n_{d}\right)
$$

Now, for $1 \leq j \leq k-1$, we introduce the notation

$$
\gamma_{k, j}:=\left(\gamma, \mathbf{0}_{k-j}, \mathbf{1}_{j-1}\right) \quad \text { and } \quad \mathbf{n}_{k, j}:=\left(\mathbf{n}_{d-k+1}, \mathbf{0}_{k-j}, \overleftarrow{\mathbf{n}}_{d+2-j}\right)
$$

Using these notations, we can write the element $Q_{n-j} \in H_{n-j}^{d}\left(W_{\gamma, \mathbf{0}_{k-j}, \mathbf{1}_{j}}\right)$ as

$$
Q_{n-j}(x)=x^{-\gamma_{k, j}}(1-|x|)^{-1} \frac{\partial^{n-j}}{\partial x^{\mathbf{n}_{k, j}}}\left\{x^{\gamma_{k, j}+\mathbf{n}_{k, j}}(1-|x|)^{\left|\mathbf{n}_{k, j}\right|+1}\right\},
$$

where $\left|\mathbf{n}_{k, j}\right|=n-j$. Consequently, our $f$ is of the form

$$
f(x)=\mathbf{x}_{d-k+1}^{-\gamma} \frac{\partial^{n-j}}{\partial x^{\mathbf{n}_{k, j}}}\left\{x^{\gamma_{k, j}+\mathbf{n}_{k, j}}(1-|x|)^{\left|\mathbf{n}_{k, j}\right|+1}\right\} .
$$

The expression shows that $f(x)=0$ whenever one of $x_{d+2-j}, \ldots, x_{d}, x_{d+1}$ is zero. It follows readily that $[f, g]_{4}=0$, since the indices of $\left\{x_{d+2-j}, \ldots, x_{d}, x_{d+1}\right\}$ are in $\mathbf{m}_{k}^{+}$for $1 \leq j \leq k-1$. The variables in $\left\{x_{d+2-j}, \ldots, x_{d}\right\}$ whose indices are in $\mathbf{m}_{k}$ appear as product factors in $\frac{\partial f}{\partial x_{i}}$, so that $\frac{\partial f}{\partial x_{i}}$ vanishes on the face $T_{\mathbf{m}_{k}}^{d-k+1}$ except when $j=1$; consequently $[f, g]_{3}=0$ except when $j=1$. If $j=1$ then $f(x)=x_{d+1} Q_{n-1}(x)$, where $Q_{n-1}(x) \in H_{n-1}^{d}\left(W_{\gamma, \mathbf{0}_{k-1}, 1}\right)$, so that an integration by parts shows that

$$
[f, g]_{3}=-\sum_{i=1}^{d-k+1} \lambda_{i} \int_{T_{\mathbf{m}_{k}}^{d-k+1}} \mathbf{x}_{d+1-k}^{\gamma} f(x)\left\{\left(\gamma_{i}+1\right) \frac{\partial g}{\partial x_{i}}+x_{i} \frac{\partial^{2} g}{\partial x_{i}{ }^{2}}\right\} d \mathbf{x}_{d-k+1} .
$$

Since $f=(1-|x|) Q_{n-1}(x)$ and from Lemma 2.10, $\left.Q_{n-1}\right|_{T_{\mathbf{m}_{k}}^{d-k+1}} \in \mathcal{V}_{n-1}^{d-k+1}\left(W_{\gamma, 1}\right)$, it follows that $[f, g]_{3}=0$ in the case of $j=1$ as the term in the curly bracket is a polynomial of degree at most $n-2$. Thus, $[f, g]_{3}=0$ for $1 \leq j \leq k-1$. 
Next we consider $[f, g]_{1}$. For convenience, we introduce the following notation: For a fixed $j$ with $1 \leq j \leq k-1$, define

$$
\mathbf{m}_{k, j}:=\{d-k+2, \ldots, d-j+1\} \quad \text { and } \quad \mathbf{m}_{k, j}^{c}:=\{d-j+2, \ldots, d\}
$$

so that $\mathbf{m}_{k}=\mathbf{m}_{k, j} \cup \mathbf{m}_{k, j}^{c}$. In particular, if $j=1$, then $\mathbf{m}_{k, j}=\mathbf{m}_{k}$ and $\mathbf{m}_{k, j}^{c}=\emptyset$. If $n<k$, then $\left|\mathbf{m}_{k}\right|=k-1>n-1$ and $\frac{\partial^{k-1} g}{\partial x^{\mathbf{m}_{k}}}=0$, so that $[f, g]_{1}=0$. If $n \geq k$, then $\left|\mathbf{m}_{k}\right| \leq n-1$. Assume first $2 \leq j \leq k-1$. Then $\left|\mathbf{m}_{k, j}^{c}\right| \geq 1$. We perform integration by parts $\left|\mathbf{m}_{k, j}^{c}\right|$ times, once for each of the variables $x_{d+2-j}, \ldots, x_{d}$, which appear as product factors in $f$, to obtain

$$
\begin{aligned}
{[f, g]_{1} } & =\int_{T^{d}} \frac{\partial^{k-1} f}{\partial x^{\mathbf{m}_{k}}} \frac{\partial^{k-1} g}{\partial x^{\mathbf{m}_{k}}}(1-|x|)^{\left|\mathbf{m}_{k}\right|-1} \mathbf{x}_{d+1-k}^{\gamma} d x \\
& =(-1)^{\left|\mathbf{m}_{k, j}^{c}\right|} \int_{T^{d}} \mathbf{x}_{d+1-k}^{\gamma} \frac{\partial^{\left|\mathbf{m}_{k, j}\right|} f}{\partial x^{\mathbf{m}_{k, j}}} \frac{\partial^{\left|\mathbf{m}_{k, j}^{c}\right|}}{\partial x^{\mathbf{m}_{k, j}^{c}}}\left\{\frac{\partial^{k-1} g}{\partial x^{\mathbf{m}_{k}}}(1-|x|)^{\left|\mathbf{m}_{k}\right|-1}\right\} d x .
\end{aligned}
$$

Using the product rule, for a generic function $h$,

$$
\frac{\partial}{\partial x}\left\{h(x)(1-x)^{n}\right\}=(1-x)^{n-1}\left\{(1-x) h^{\prime}(x)-n h(x)\right\},
$$

it is easy to see that we can write

$$
\frac{\partial^{\left|\mathbf{m}_{k, j}^{c}\right|}}{\partial x^{\mathbf{m}_{k, j}^{c}}}\left\{\frac{\partial^{k-1} g}{\partial x^{\mathbf{m}_{k}}}(1-|x|)^{\left|\mathbf{m}_{k}\right|-1}\right\}=(1-|x|)^{\left|\mathbf{m}_{k, j}\right|-1} h_{n-\left|\mathbf{m}_{k}\right|-1}(x)
$$

where $h_{n-\left|\mathbf{m}_{k}\right|-1}$ is a polynomial of degree $\left(n-\left|\mathbf{m}_{k}\right|-1\right)$. Consequently, we have

$$
[f, g]_{1}=(-1)^{\left|\mathbf{m}_{k, j}^{c}\right|} \int_{T^{d}} \mathbf{x}_{d+1-k}^{\gamma} \frac{\partial^{\left|\mathbf{m}_{k, j}\right|} f}{\partial x^{\mathbf{m}_{k, j}}}\left\{(1-|x|)^{\left|\mathbf{m}_{k, j}\right|-1} h_{n-\left|\mathbf{m}_{k}\right|-1}(x)\right\} d x .
$$

Now, taking derivative of $f$ in (4.7) with respect to variables whose indices are in $\mathbf{m}_{k, j}$, we obtain, as these derivatives only apply to the factor $(1-|x|)$ in $f$, that

$$
\frac{\partial^{\left|\mathbf{m}_{k, j}\right|} f}{\partial \mathbf{x}_{k, j}^{\mathbf{m}_{k, j}}}=c_{k, j} x_{d+2-j} \cdots x_{d}(1-|x|)^{-\left(\left|\mathbf{m}_{k, j}\right|-1\right)} P_{\mathbf{n}}^{\left(\gamma, \mathbf{0}_{k-j}, \mathbf{1}_{j-1},-\left(\left|\mathbf{m}_{k, j}\right|-1\right)\right)}(x),
$$

where $c_{k, j}$ is a constant, $\mathbf{x}_{k, j}=\left(x_{d-k+2}, \ldots, x_{d-j+1}\right)$ and $\mathbf{n}=\mathbf{n}_{k, j}$. Substituting it into the expression for $[f, g]_{1}$, we obtain

$$
\begin{aligned}
{[f, g]_{1}=} & (-1)^{\left|\mathbf{m}_{k, j}^{c}\right|} c_{k, j} \int_{T^{d}} \mathbf{x}_{d+1-k}^{\gamma} x_{d+2-j} \cdots x_{d} \\
& \times P_{\mathbf{n}}^{\left(\gamma, \mathbf{0}_{k-j}, \mathbf{1}_{j-1},-\left(\left|\mathbf{m}_{k, j}\right|-1\right)\right)}(x) h_{n-\left|\mathbf{m}_{k}\right|-1}(x) d x .
\end{aligned}
$$

Since $\left|\mathbf{m}_{k, j}^{c}\right|=j-1$ and $|\mathbf{n}|=n-j$, we can write $|\mathbf{n}|=n-\left|\mathbf{m}_{k, j}^{c}\right|-1$. As $\left|\mathbf{m}_{k}\right|=\left|\mathbf{m}_{k, j}\right|+\left|\mathbf{m}_{k, j}^{c}\right|$, we see that

$$
\operatorname{deg} P_{\mathbf{n}}^{\left(\gamma, \mathbf{0}_{k-j}, \mathbf{1}_{j-1},-\left(\left|\mathbf{m}_{k, j}\right|-1\right)\right)}>\left(\left|\mathbf{m}_{k, j}\right|-1\right)+\operatorname{deg} h_{n-\left|\mathbf{m}_{k}\right|-1},
$$

so that, by Lemma $2.3[f, g]_{1}=0$ for all $g \in \Pi_{n-1}^{d}$ when $2 \leq j \leq k-1$. In the case $j=1,\left|\mathbf{m}_{k, j}^{c}\right|=0$ and there is no need for integration by parts in $[f, g]_{1}$. In this case, (4.9) becomes

$$
\frac{\partial^{\left|\mathbf{m}_{k}\right| f}}{\partial x^{\mathbf{m}_{k}}}=c_{k, 1}(1-|x|)^{-\left(\left|\mathbf{m}_{k}\right|-1\right)} P_{\mathbf{n}}^{\left(\gamma, \mathbf{0}_{k-1},-\left(\left|\mathbf{m}_{k}\right|-1\right)\right)}(x)
$$


and $|\mathbf{n}|=n-1$, so that $[f, g]_{1}$ becomes

$$
\begin{aligned}
{[f, g]_{1} } & =\int_{T^{d}} \frac{\partial^{k-1} f}{\partial x^{\mathbf{m}_{k}}} \frac{\partial^{k-1} g}{\partial x^{\mathbf{m}_{k}}}(1-|x|)^{\left|\mathbf{m}_{k}\right|-1} \mathbf{x}_{d+1-k}^{\gamma} d x \\
& =c_{k, 1} \int_{T^{d}} \mathbf{x}_{d+1-k}^{\gamma} P_{\mathbf{n}}^{\left(\gamma, \mathbf{0}_{k-1},-\left(\left|\mathbf{m}_{k}\right|-1\right)\right)}(x) \frac{\partial^{k-1} g}{\partial x^{\mathbf{m}_{k}}} d x .
\end{aligned}
$$

Since $|\mathbf{n}|>\left(\left|\mathbf{m}_{k}\right|-1\right)+\operatorname{deg} \frac{\partial^{k-1} g}{\partial x^{\mathbf{m}} k}$ for $g \in \Pi_{n-1}^{d}$, it follows from Lemma 2.3 that $[f, g]_{1}=0$. This completes the proof that $[f, g]_{1}=0$.

Next we deal with $[f, g]_{2}=0$. In the proof, let $I=I_{1} \cup I_{2}, I_{1} \subset \mathbf{m}_{k, j}$ and $I_{2} \subset \mathbf{m}_{k, j}^{c}$. Firstly, assume that $I_{2}$ is a proper subset of $\mathbf{m}_{k, j}^{c}$ for $2 \leq j \leq k-1$. Then at least one of the variables in $\left\{x_{d+2-j}, \ldots, x_{d}\right\}$ whose indices are in $\mathbf{m}_{k, j}^{c}$ appears as a product factor in $\frac{\partial^{i} f}{\partial x^{I}}$, so that the function $\frac{\partial^{i} f}{\partial x^{T}}$ vanishes on the face $T_{I^{c}}^{d-k+i+1}$ and, consequently, $[f, g]_{2}=0$. Secondly, assume that $I_{2}=\mathbf{m}_{k, j}^{c}$. Then $\left|I_{2}\right|=\left|\mathbf{m}_{k, j}^{c}\right|=j-1$ for $1 \leq j \leq k-1$ and the sum over $i$ in $[f, g]_{2}$ starts at $i=j-1$ if $j>1$, so that we can write

$$
[f, g]_{2}=\sum_{i=\max \{1, j-1\}}^{k-2} \sum_{\substack{I_{1} \subset \mathbf{m}_{k, j} \\ I_{2}=\mathbf{m}_{k, j}^{c} \\|I|=i}} \lambda_{I}[f, g]_{2, i, I},
$$

where

$$
[f, g]_{2, i, I}:=\int_{T_{I^{c}}^{d-k+i+1}} \frac{\partial^{i} f}{\partial x^{I}} \frac{\partial^{i} g}{\partial x^{I}}(1-|x|)^{|I|-1} \mathbf{x}_{d+1-k}^{\gamma} d \mathbf{x}_{d-k+1} d \mathbf{x}_{I} .
$$

For $j \geq 1,\left|I_{2}\right|=j-1 \geq 0$. If $j>1$, we apply integration by parts $\left|I_{2}\right|$ times over the variables $x_{d+2-j}, \ldots, x_{d}$, which appear as product factors in $f$ by (4.7), to obtain

$$
\begin{aligned}
{[f, g]_{2, i, I} } & :=\int_{T_{I^{c}}^{d-k+i+1}} \frac{\partial^{i} f}{\partial x^{I}} \frac{\partial^{i} g}{\partial x^{I}}(1-|x|)^{|I|-1} \mathbf{x}_{d+1-k}^{\gamma} d \mathbf{x}_{d-k+1} d \mathbf{x}_{I} \\
& =(-1)^{\left|I_{2}\right|} \int_{T_{I^{c}}^{d-k+i+1}} \mathbf{x}_{d+1-k}^{\gamma} \frac{\partial^{\left|I_{1}\right|} f}{\partial x^{I_{1}}} \frac{\partial^{\left|I_{2}\right|}}{\partial x^{I_{2}}}\left\{\frac{\partial^{|I|} g}{\partial x^{I}}(1-|x|)^{|I|-1}\right\} d \mathbf{x}_{d-k+1} d \mathbf{x}_{I}
\end{aligned}
$$

for $i=|I| \geq j-1$. If $I_{1} \neq \emptyset$ then, using the product rule (4.8), we can write

$$
\frac{\partial^{\left|I_{2}\right|}}{\partial x^{I_{2}}}\left\{\frac{\partial^{|I|} g}{\partial x^{I}}(1-|x|)^{|I|-1}\right\}=(1-|x|)^{\left|I_{1}\right|-1} h_{n-|I|-1},
$$

where $h_{n-|I|-1}$ is a polynomial of degree $(n-|I|-1)$, we further deduce that

$$
[f, g]_{2, i, I}=(-1)^{\left|I_{2}\right|} \int_{T_{I^{c}}^{d-k+i+1}} \mathbf{x}_{d+1-k}^{\gamma} \frac{\partial^{\left|I_{1}\right|} f}{\partial x^{I_{1}}}\left\{(1-|x|)^{\left|I_{1}\right|-1} h_{n-|I|-1}\right\} d \mathbf{x}_{d-k+1} d \mathbf{x}_{I},
$$

which also holds if $j=1$, for which there is no need to take derivatives as $\left|I_{2}\right|=0$. Furthermore, similar to (4.9), if we take derivatives of (4.7) with respect to variables whose indices are in $I_{1}$, then the derivatives apply only on the factor $(1-|x|)$, so that

$$
\frac{\partial^{\left|I_{1}\right|} f}{\partial x^{I_{1}}}=d_{k, j} x_{d+2-j} \cdots x_{d}(1-|x|)^{-\left(\left|I_{1}\right|-1\right)} P_{\mathbf{n}}^{\left(\gamma, \mathbf{0}_{k-j}, \mathbf{1}_{j-1},-\left(\left|I_{1}\right|-1\right)\right)}(x),
$$


where $\mathbf{n}=\mathbf{n}_{k, j}$ and $d_{k, j}$ is a constant, which allows us to write

$$
\begin{aligned}
{[f, g]_{2, i, I}=} & (-1)^{\left|I_{2}\right|} d_{k, j} \int_{T_{I^{c}}^{d-k+i+1}} \mathbf{x}_{d+1-k}^{\gamma} x_{d+2-j} \cdots x_{d} \\
& \times P_{\mathbf{n}}^{\left(\gamma, \mathbf{0}_{k-j}, \mathbf{1}_{j-1},-\left(\left|I_{1}\right|-1\right)\right)}(x) h_{n-|I|-1}(x) d \mathbf{x}_{d-k+1} d \mathbf{x}_{I} .
\end{aligned}
$$

By Lemma 2.10, the restriction of $P_{\mathbf{n}}^{\left(\gamma, \mathbf{0}_{k-j}, \mathbf{1}_{j-1},-\left(\left|I_{1}\right|-1\right)\right)}(x)$ on the face $T_{I^{c}}^{d-k+i+1}$ is the polynomial $P_{\mathbf{n}}^{\left(\gamma, \mathbf{0}_{i-j+1}, \mathbf{1}_{j-1},-\left(\left|I_{1}\right|-1\right)\right)}\left(\mathbf{x}_{d-k+1}, \mathbf{x}_{I}\right)$ in the $(d-k+i+1)$-variables of $\mathbf{x}_{d-k+1}$ and $\mathbf{x}_{I}$ on $T_{I^{c}}^{d-k+i+1}$. Since $\left|I_{2}\right|=j-1$ and $|\mathbf{n}|=n-j$, we have $|\mathbf{n}|>\left(\left|I_{1}\right|-1\right)+\operatorname{deg} h$ and, consequently, by Lemma 2.3, that $[f, g]_{2, i, I}=0$. As a result, we have shown that the first term of $[f, g]_{2}$ in (4.10) is zero for $1 \leq j \leq k-2$ and $I_{1} \neq \emptyset$.

If, however, $I_{1}=\emptyset$, then $I=I_{2}=\mathbf{m}_{k, j}^{c}$, so that $i=|I|=j-1$ and, in particular, as $i \geq 1, j \geq 2$. We apply integration by parts $(|I|-1)$ times over the variables $x_{d+3-j}, \ldots, x_{d}$ to obtain

$$
[f, g]_{2, j-1, I}=(-1)^{|I|-1} \int_{T_{I c}^{d-(k-j)}} \mathbf{x}_{d+1-k}^{\gamma} \frac{\partial f}{\partial x_{d+2-j}} h_{n-|I|-1} d \mathbf{x}_{d-k+1} d \mathbf{x}_{I},
$$

where $h_{n-|I|-1}$ is the polynomial of degree $(n-|I|-1)$ given by

$$
h_{n-|I|-1}=\frac{\partial^{|I|-1}}{\partial x_{d+3-j} \cdots \partial x_{d}}\left\{\frac{\partial^{|I|} g}{\partial x^{I}}(1-|x|)^{|I|-1}\right\} .
$$

Now, if $j \geq 3$, taking derivative of (4.7) with respect to $x_{d+2-j}$ gives

$$
\frac{\partial f}{\partial x_{d+2-j}}=x_{d+3-j} \cdots x_{d} P_{\mathbf{n}}^{\left(\gamma, \mathbf{0}_{k-j+1}, \mathbf{1}_{j-2}, 0\right)}(x),
$$

where $\mathbf{n}=\mathbf{n}_{k, j}+e_{d+2-j}$ and $|\mathbf{n}|=n-|I|$, from which follows immediately that

$$
\begin{aligned}
{[f, g]_{2, j-1, I}=} & (-1)^{|I|-1} \int_{T_{I^{c}}^{d-(k-j)}} \mathbf{x}_{d+1-k}^{\gamma} x_{d+3-j} \cdots x_{d} \\
& \times P_{\mathbf{n}}^{\left(\gamma, \mathbf{0}_{k-j+1}, \mathbf{1}_{j-2}, 0\right)}(x) h_{n-|I|-1}(x) d \mathbf{x}_{d-k+1} d \mathbf{x}_{I} .
\end{aligned}
$$

Since $|\mathbf{n}|=n-|I|$ and, by Lemma 2.10 $\left.P_{\mathbf{n}}^{\left(\gamma, \mathbf{0}_{k-j+1}, \mathbf{1}_{j-2}, 0\right)}(x)\right|_{T_{I^{c}}^{d-(k-j)}}$ is an element of $\mathcal{V}_{n-|I|}^{d-(k-j)}\left(W_{\gamma, 0, \mathbf{1}_{j-2}, 0}\right)$ in the variables $\left(\mathbf{x}_{d-k+1}, \mathbf{x}_{I}\right)$, it follows from the orthogonality of $\mathcal{V}_{n-|I|}^{d-(k-j)}\left(W_{\gamma, 0, \mathbf{1}_{j-2}, 0}\right)$ that $[f, g]_{2, j-1, I}=0$ for $j \geq 3$. If, however, $j=2$, then $I_{2}=\{d\}$ and $[f, g]_{2, j-1, I}$ for $j=2$ becomes

$$
[f, g]_{2,1, I}=\int_{T_{\{d-k+2, \ldots, d-1\}}^{d-k+2}} \mathbf{x}_{d+1-k}^{\gamma} \frac{\partial f}{\partial x_{d}} \frac{\partial g}{\partial x_{d}} d \mathbf{x}_{d-k+1} d x_{d} .
$$

By (4.12) with $j=2, \frac{\partial f}{\partial x_{d}}=P_{\mathbf{n}}^{\left(\gamma, \mathbf{0}_{k-1}, 0\right)}(x)$, which, by Lemma 2.10, is an element in $\mathcal{V}_{n-1}^{d-k+2}\left(W_{\gamma, 0,0}\right)$ when restricted to the face $T_{\{d-k+2, \ldots, d-1\}}^{d-k+2}$. Consequently, since $|\mathbf{n}|=n-1$ in this case, it follows from the orthogonality of $\mathcal{V}_{n-1}^{d-k+2}\left(W_{\gamma, 0,0}\right)$ that $[f, g]_{2,1, I}=0$. Thus, we have establish that $[f, g]_{2, i, I}=0$ whenever $j-1 \leq i \leq k-2$ and $j \geq 2$.

Putting these together, we have proved that $\langle f, g\rangle_{\gamma,-\mathbf{1}_{k}}=0$ for all $g \in \Pi_{n-1}^{d}$. Consequently, the proof of Case 2.2 is completed. 
Case 2.3. $f$ is an element of $x_{j, k}(\sigma) H_{n-j}^{d}\left(W_{\gamma,\left(\mathbf{0}_{k-j}, \mathbf{1}_{j}\right) \sigma}\right)$, where $1 \leq j \leq k-1$ and $\sigma \in \mathcal{G}_{k}$, and $x_{j, k}$ does not contain the variable $x_{d+1}$.

As in the Case 2.2, it is sufficient to consider $\sigma=i d$. Since $x_{j, k}$ does not contain $x_{d+1}$, we can assume that

$$
x_{j, k}=x_{d+2-j} \cdots x_{d} \quad \text { and } \quad f(x)=x_{d+2-j} \cdots x_{d} Q_{n-j+1}(x)
$$

for $2 \leq j \leq k$, where $Q_{n-j+1} \in H_{n-j+1}^{d}\left(W_{\gamma, \mathbf{0}_{k-j}, \mathbf{1}_{j-1}, 0}\right)$. From Definition 2.9. $x_{S_{k-j+1}}=\left\{1-|x|, x_{d-k+2}, \ldots, x_{d-j+1}\right\}$ and then

$$
\begin{array}{r}
H_{n}^{d}\left(W_{\gamma, \mathbf{0}_{k-j}, \mathbf{1}_{j-1}, 0}\right)=\left\{P_{\mathbf{n}}^{\left(\mathbf{0}_{k-j+1}, \mathbf{1}_{j-1}, \gamma\right)}\left(x_{S_{k-j+1}}, \overleftarrow{\mathbf{x}}_{d-j+2}, \mathbf{x}_{d-k}\right):\right. \\
\left.\mathbf{n}=\left(\mathbf{0}, \mathbf{n}_{k, j}\right),|\mathbf{n}|=n, \mathbf{n}_{k, j} \in \mathbb{N}_{0}^{d-k+j-1}\right\} .
\end{array}
$$

From Definition 2.4 we can explicitly write the Rodrigue basis of this space. To keep the notation compact, we use following notations in this case of the proof,

$$
\mathbf{x}_{k, j}:=\left(\overleftarrow{\mathbf{x}}_{d-j+2}, \mathbf{x}_{d-k}\right) \quad \text { and } \quad \gamma_{k, j}:=\left(\mathbf{1}_{j-1}, \gamma_{d-k}\right)
$$

In case 2 of Definition 2.4 taking

$$
\begin{aligned}
& x_{i_{2}}=x_{d-k+2}, \ldots, x_{i_{k}}=x_{d}, \quad x_{i_{k+1}}=x_{1}, \ldots, x_{i_{d+1}}=x_{d-k+1} \\
& \mathbf{n}=\left(\mathbf{n}_{k-j+1}, \mathbf{n}_{k, j}\right) \quad \text { with } \quad \mathbf{n}_{k-j+1}=\left(n_{1}, \ldots, n_{k-j+1}\right), \quad \mathbf{n}_{k, j} \in \mathbb{N}_{0}^{d-k+j-1},
\end{aligned}
$$

then we can write

$$
\begin{aligned}
& P_{\mathbf{n}}^{\left(\mathbf{0}_{k-j+1}, \boldsymbol{\gamma}_{k, j}, \gamma_{d-k+1}\right)}\left(x_{S_{k-j+1}}, \mathbf{x}_{k, j}\right) \\
& \quad=(-1)^{n_{1}} \mathbf{x}_{k, j}^{-\gamma_{k, j}} x_{d-k+1}^{-\gamma_{d-k+1}} \frac{\partial^{|\mathbf{n}|}}{\partial \mathbf{u}_{\mathbf{k}, \mathbf{j}}^{\mathbf{n}}}\left\{x_{S_{k-j+1}}^{\mathbf{n}_{k-j+1}} \mathbf{x}_{k, j}^{\gamma_{k, j}+\mathbf{n}_{k, j}} x_{d-k+1}^{\gamma_{d-k+1}+|\mathbf{n}|}\right\},
\end{aligned}
$$

where $\partial \mathbf{u}_{\mathbf{k}, \mathbf{j}}=\left(\partial_{d-k+1}, \partial_{d-k+2, d-k+1}, \ldots, \partial_{d, d-k+1}, \partial_{1, d-k+1}, \ldots, \partial_{d-k, d-k+1}\right)$ and recall that $\partial_{i, j}=\partial_{i}-\partial_{j}$. Since the first $(k-j+1)$ components of $\mathbf{n}$ are zero, in the definition of $H_{n}^{d}\left(W_{\gamma, \mathbf{0}_{k-j}, \mathbf{1}_{j-1}, 0}\right)$, we can write $Q_{n-j+1} \in H_{n-j+1}^{d}\left(W_{\gamma, \mathbf{0}_{k-j}, \mathbf{1}_{j-1}, 0}\right)$ as

$$
Q_{n-j+1}(x)=\mathbf{x}_{k, j}^{-\gamma_{k, j}} x_{d-k+1}^{-\gamma_{d-k+1}} \frac{\partial^{\left|\mathbf{n}_{k, j}\right|}}{\partial \mathbf{y}_{\mathbf{k}, j}^{\mathbf{n}_{k, j}}}\left\{\mathbf{x}_{k, j}^{\gamma_{k, j}+\mathbf{n}_{k, j}} x_{d-k+1}^{\gamma_{d-k+1}+\left|\mathbf{n}_{k, j}\right|}\right\},
$$

where $\partial \mathbf{y}_{\mathbf{k}, \mathbf{j}}=\left(\partial_{d-j+2, d-k+1}, \ldots, \partial_{d, d-k+1}, \partial_{1, d-k+1}, \ldots, \partial_{d-k, d-k+1}\right)$. Hence, we conclude that

$$
f(x)=\mathbf{x}_{d-k+1}^{-\gamma_{d-k+1}} \frac{\partial^{n-j+1}}{\partial \mathbf{y}_{\mathbf{k}, \mathbf{j}}^{\mathbf{n}_{k, j}}}\left\{\mathbf{x}_{k, j}^{\gamma_{k, j}+\mathbf{n}_{k, j}} x_{d-k+1}^{\left|\mathbf{n}_{k, j}\right|+\gamma_{d-k+1}}\right\},
$$

where $\left|\mathbf{n}_{k, j}\right|=n-j+1$.

This expression of $f$ shows that $f(x)=0$ whenever one of $x_{d+2-j}, \ldots, x_{d}$ is zero. It follows readily that $[f, g]_{4}=0$, since the indices of $\left\{x_{d+2-j}, \ldots, x_{d}\right\}$ are among the variables whose indices are in $\mathbf{m}_{k}^{+}$for $2 \leq j \leq k$, so that the restriction of $f$ on the face $T_{\mathbf{m}_{k}^{+}}^{d-k}$ is zero. Furthermore, $[f, g]_{3}=0$ for the same reason. Since the variables in $\left\{x_{d-k+2}, \ldots, x_{d-j+1}\right\}, 2 \leq j \leq k-1$, do not appear in $f, \frac{\partial^{\left|\mathbf{m}_{k}\right|} f}{\partial x^{\mathbf{m}_{k}}}=0$, so that $[f, g]_{1}=0$ except for $j=k$. We now consider the case of $j=k$. In this case, by (4.13) with $j=k$,

$$
f(x)=\mathbf{x}_{d-k+1}^{-\gamma_{d-k+1}} \frac{\partial^{n-k+1}}{\partial \mathbf{y}_{\mathbf{k}, \mathbf{k}}^{\mathbf{n}_{k, k}}}\left\{\mathbf{x}_{k, k}^{\gamma_{k, k}+\mathbf{n}_{k, k}} x_{d-k+1}^{\left|\mathbf{n}_{k, k}\right|+\gamma_{d-k+1}}\right\}
$$


where $\left|\mathbf{n}_{k, k}\right|=n-k+1$. Applying integration by parts $\left|\mathbf{m}_{k}\right|-1$ times over the variables $x_{d-k+3}, \ldots, x_{d}$, which are product factors in $f$, we obtain

$$
[f, g]_{1}=(-1)^{\left|\mathbf{m}_{k}\right|-1} \int_{T^{d}} \mathbf{x}_{d+1-k}^{\gamma} \frac{\partial f}{\partial x_{d-k+2}} \frac{\partial^{\left|\mathbf{m}_{k}\right|-1}}{\partial \mathbf{x}_{d-k+3}}\left\{\frac{\partial^{d-k} g}{\partial x^{\mathbf{m}_{k}}}(1-|x|)^{\left|\mathbf{m}_{k}\right|-1}\right\} d x
$$

where $\frac{\partial^{\left|\mathbf{m}_{k}\right|-1}}{\partial \mathbf{x}_{d-k+3}}=\frac{\partial^{\left|\mathbf{m}_{k}\right|-1}}{\partial x_{d-k+3} \cdots \partial x_{d}}$. Taking derivative of $f$ in (4.14) with respect to $x_{d-k+2}$, we obtain

$$
\frac{\partial f}{\partial x_{d-k+2}}=c x_{d-k+3} \cdots x_{d} P_{\mathbf{n}}^{\left(0,0, \mathbf{1}_{k-2}, \gamma\right)}\left(x_{S_{k-j+1}}, \mathbf{x}_{k, j}\right)
$$

where $c$ is a constant and $\mathbf{n}=\left(\mathbf{0}, \mathbf{n}_{k, k}\right)$. Since $\frac{\partial^{\left|\mathbf{m}_{k}\right|-1}}{\partial \mathbf{x}_{d-k+3}}\left\{\frac{\partial^{d-k} g}{\partial x^{\mathbf{m}_{k}}}(1-|x|)^{\left|\mathbf{m}_{k}\right|-1}\right\}$ is a polynomial of degree $n-\left|\mathbf{m}_{k}\right|-1$ and $|\mathbf{n}|=n-k+1=n-\left|\mathbf{m}_{k}\right|$, we deduce that $[f, g]_{1}=0$ form the fact that $P_{\mathbf{n}}^{\left(0,0, \mathbf{1}_{k-2}, \gamma\right)} \in \mathcal{V}_{n-\left|\mathbf{m}_{k}\right|}^{d}\left(W_{\left(\gamma, 0, \mathbf{1}_{k-2}, 0\right)}\right)$. Thus, $[f, g]_{1}=0$ for $2 \leq j \leq k$.

It remains to consider $[f, g]_{2}$. Firstly, assume that $I_{2}$ is a proper subset of $\mathbf{m}_{k, j}^{c}$ for $2 \leq j \leq k$. Since at least one of the variables in $\left\{x_{d+2-j}, \ldots, x_{d}\right\}$ whose indices are in $\mathbf{m}_{k, j}^{c}$ appears as a product factor in $\frac{\partial^{i} f}{\partial x^{I}}$, the function $\frac{\partial^{i} f}{\partial x^{T}}$ vanishes on the face $T_{I^{c}}^{d-k+i+1}$ so that $[f, g]_{2}=0$. Secondly, assume that $I_{2}=\mathbf{m}_{k, j}^{c}=\{d-j+2, \ldots, d\}$. Then $\left|I_{2}\right|=\left|\mathbf{m}_{k, j}^{c}\right|=j-1$ for $2 \leq j \leq k-1$. We do not need to consider the case $j=k$ when $I_{2}=\mathbf{m}_{k, j}^{c}$, since if $j=k$ then $\mathbf{m}_{k, j}=\emptyset$ and $\mathbf{m}_{k}=\mathbf{m}_{k, j}^{c}=I_{2}$, so that $I=\mathbf{m}_{k}$, which contradicts to the fact that $I$ is a proper subset of $\mathbf{m}_{k}$ in $[f, g]_{2}$. Thus, we only need to consider $2 \leq j \leq k-1$, for which the summation over $i$ in $[f, g]_{2}$ starts at $i=j-1$, so that we can write

$$
[f, g]_{2}=\sum_{i=j-1}^{k-2} \sum_{\substack{I_{1} \subset \mathbf{m}_{k, j} \\ I_{2}=\mathbf{m}_{k, j} \\|I|=i}} \lambda_{I}[f, g]_{2, i, I},
$$

where $[f, g]_{2, i, I}$ are given as in (4.10). Since the variables $x_{d-k+2}, \ldots, x_{d-j+1}$ whose indices are in $\mathbf{m}_{k, j}$ for $2 \leq j \leq k-1$ do not appear in $f, \frac{\partial^{\left|I_{1}\right|_{f}}}{\partial x^{I_{1}}}=0$ and, consequently, $[f, g]_{2, i, I}=0$ if $i \geq j$. For $i=j-1,\left|I_{2}\right|=|I|=j-1$ and $I_{2}=I=\{d-j+2, \ldots, d\}$; in particular, $I_{2}^{c}=I^{c}=\{d-k+2, \ldots, d-j+1\}$. Applying integration by parts $\left|I_{2}\right|-1$ times over the variables $x_{d+3-j}, \ldots, x_{d}$, which appeared as product factors in $f$, we find

$$
[f, g]_{2, j-2, I}=(-1)^{j} \int_{T_{I^{c}}} \mathbf{x}_{d+1-k}^{\gamma} \frac{\partial f}{\partial x_{d+2-j}} h_{n-j}(x) d \mathbf{x}_{d-k+1} d \mathbf{x}_{I},
$$

where $h_{n-j}$ is a polynomial of degree $n-j$ given by

$$
h_{n-j}(x)=\frac{\partial^{j-2}}{\partial \mathbf{x}_{d+3-j}}\left\{\frac{\partial^{j-1} g}{\partial x^{I_{2}}}(1-|x|)^{j-2}\right\} .
$$

Now, taking derivative of $f$ in (4.13) with respect to $x_{d+2-j}$, we obtain

$$
\frac{\partial f}{\partial x_{d+2-j}}=d x_{d-j+3} \cdots x_{d} P_{\mathbf{n}}^{\left(\mathbf{0}_{k-j+2}, \mathbf{1}_{j-2}, \gamma\right)}\left(x_{S_{k-j+1}}, \mathbf{x}_{k, j}\right)
$$

where $d$ is a constant and $\mathbf{n}=\left(\mathbf{0}, \mathbf{n}_{k, j}\right)$. The polynomial $P_{\mathbf{n}}^{\left(\mathbf{0}_{k-j+2}, \mathbf{1}_{j-2}, \gamma\right)}\left(x_{S_{k-j+1}}, \mathbf{x}_{k, j}\right)$ is, by Lemma 2.10 an element of $\mathcal{V}_{n-j+1}^{d-(k-j)}\left(W_{\gamma, 0, \mathbf{1}_{j-2}, 0}\right)$ when restricted to the face 
$T_{\{d-k+2, \ldots, d-j+1\}}^{d-(k-j)}$. Consequently, since $|\mathbf{n}|=n-j+1$ and $h$ is a polynomial of degree $n-j,[f, g]_{2, j-1, I}=0$ follows from the orthogonality of $\mathcal{V}_{n-j+1}^{d-(k-j)}\left(W_{\gamma, 0, \mathbf{1}_{j-2}, 0}\right)$. Hence, $[f, g]_{2}=0$ for all $g \in \Pi_{n-1}^{d}$.

Putting these together, we have proved that $\langle f, g\rangle_{\gamma,-\mathbf{1}_{k}}=0$ for all $g \in \Pi_{n-1}^{d}$. Consequently, the proof of Case 2.3 is completed.

Case 2.4. $f \in H_{n}^{d}\left(W_{\gamma, \mathbf{0}_{k}}\right)$. From Definition 2.9, we can assume

$$
f(x)=P_{\mathbf{n}}^{(\mathbf{0}, \gamma)}\left(x_{d-k+2}, \ldots, x_{d+1}, \mathbf{x}_{d-k}\right), \quad|\mathbf{n}|=n,
$$

where $x_{d+1}=1-|x|$ and $\mathbf{n}=\left(\mathbf{0}, \mathbf{n}_{d-k}\right), \mathbf{n}_{d-k}=\left(n_{1}, \ldots, n_{d-k}\right)$. Since the first $k$ components of $\mathbf{n}$ are zero, the Rodrigue formula (2.9) shows that the variables whose indices are in $\mathbf{m}_{k}$ do not really appear in $f$. Hence, $\frac{\partial^{k-1} f}{\partial x^{\mathbf{m}} k}=0$ and $\frac{\partial^{i} f}{\partial x^{T}}=0$ for $I \subset \mathbf{m}_{k}$, from which it follows that $[f, g]_{1}=0$ and $[f, g]_{2}=0$, respectively. For the integral in $[f, g]_{3}$, we apply integration by parts to obtain

$$
[f, g]_{3}=-\sum_{j=1}^{d-k+1} \lambda_{j} \int_{T_{\mathbf{m}_{k}}^{d-k+1}} \mathbf{x}_{d+1-k}^{\gamma} f\left\{\left(\gamma_{j}+1\right) \frac{\partial g}{\partial x_{j}}+x_{j} \frac{\partial^{2} g}{\partial x_{j}^{2}}\right\} d \mathbf{x}_{d-k+1} .
$$

Since, by Lemma 2.10, $\left.f\right|_{T_{\mathbf{m}_{k}}^{d-k+1}} \in \mathcal{V}_{n}^{d-k+1}\left(W_{\gamma, 0}\right)$, and the term in the curly bracket is a polynomial of degree at most $n-2$, we conclude that $[f, g]_{3}=0$. Finally, by Lemma [2.10, $\left.f\right|_{\substack{T_{\mathbf{m}_{k}^{+}}^{d-k} \\ \text { in }}} \in \mathcal{V}_{n}^{d-k}\left(W_{\gamma}\right)$, which implies immediately that $[f, g]_{4}=0$. Thus, it follows from (4.6) that $\langle f, g\rangle_{\gamma,-\mathbf{1}_{k}}=0$ for all $g \in \Pi_{n-1}^{d}$.

Putting the four cases together, the decomposition (3.4) shows that $\langle f, g\rangle_{\gamma,-\mathbf{1}_{k}}=$ 0 for every $f \in \mathcal{U}_{n}^{d}\left(W_{\gamma,-\mathbf{1}_{k}}\right)$ and all $g \in \Pi_{n-1}^{d}$. This completes the proof of (ii) of the theorem for Case 2.

Case 3. $k=d+1$. The main terms of the inner product $\langle f, g\rangle_{-1}$ are essentially the case of $k=d+1$ of $[f, g]_{1}$ and $[f, g]_{2}$ in Case 2, hence, as shown in that case, that these two terms are zero for every $f \in \mathcal{U}_{n}^{d}\left(W_{-1}\right)$ and for all $g \in \Pi_{n-1}^{d}$. Furthermore, if $f \in x_{1} \cdots x_{d+1} \mathcal{V}_{n-d-1}^{d}\left(W_{\mathbf{1}}\right)$ then $f$ vanishes on $e_{0}, e_{1}, \ldots, e_{d}$, whereas if $f \in$ $x_{j, d+1}(\sigma) H_{n-j}^{d}\left(W_{\left(\mathbf{0}_{d+1-j}, \mathbf{1}\right) \sigma}\right)$, then $f\left(e_{0}\right)=f\left(e_{1}\right)=\ldots=f\left(e_{d}\right)=0$. Thus, every $f \in \mathcal{U}_{n}^{d}\left(W_{-1}\right)$ vanishes at all vertices, so that the last term in $\langle f, g\rangle_{-\mathbf{1}}$ is zero. Consequently, $\langle f, g\rangle_{-\mathbf{1}}=0$ for $\forall f \in \mathcal{U}_{n}^{d}\left(W_{-1}\right)$ and $\forall g \in \Pi_{n-1}^{d}$.

The proof of (ii) in Theorem 3.7 is completed.

4.5. Proof of (iii) in Theorem 3.7. We need to show that $\langle f, g\rangle_{\gamma,-1}=0$ for $f \in(1-|x|) \mathcal{V}_{n-1}^{d}\left(W_{\gamma, 1}\right)$ and $g \in H_{n}^{d}\left(W_{\gamma, 0}\right)$. The proof uses a property of $H_{n}^{d}\left(W_{\gamma, 0}\right)$, which is of interesting in itself.

By definition, $H_{n}^{d}\left(W_{\gamma, 0}\right)=H_{n,\{d+1\}}^{d}\left(W_{\gamma, 0}\right)$, that is, $S_{1}=\{d+1\}$ in the item 2 of Definition 2.9, and $\left(\gamma_{S}, \gamma_{\ell_{d}}\right)=\left(0, \gamma^{\prime}, \gamma_{\ell_{d}}\right)$, where $\gamma^{\prime}=\gamma_{S \backslash\{d+1\}}=\left(\gamma_{\ell_{1}}, \ldots, \gamma_{\ell_{d-1}}\right)$, and $x_{S}=\left(x_{S_{1}}, x_{S \backslash S_{1}}\right)=\left(x_{d+1}, x^{\prime}\right), x^{\prime}=\left(x_{\ell_{1}}, \ldots, x_{\ell_{d-1}}\right)$. Hence, by Definition 2.4 the elements of $H_{n}^{d}\left(W_{\gamma, 0}\right)$ are given by, since $n_{1}=0$,

$$
P_{\mathbf{n}}^{\left(\gamma_{S}, \gamma_{\ell_{d}}\right)}\left(x_{S}\right)=\left(x^{\prime}\right)^{-\gamma^{\prime}} x_{\ell_{d}}^{-\gamma_{\ell_{d}}} \frac{\partial^{|\mathbf{n}|}}{\partial \mathbf{x}_{S}^{\mathbf{n}^{\prime}}}\left\{\left(x^{\prime}\right)^{\gamma^{\prime}+\mathbf{n}^{\prime}} x_{\ell_{d}}^{\gamma_{\ell_{d}}+|\mathbf{n}|}\right\}
$$

where $\mathbf{n}=\left(0, \mathbf{n}^{\prime}\right)$ and $\partial \mathbf{x}_{S}=\left(\partial_{\ell_{1}, \ell_{d}}, \ldots, \partial_{\ell_{d-1}, \ell_{d}}\right)$. Consequently, the elements of $H_{n}^{d}\left(W_{\gamma, 0}\right)$ are homogeneous polynomials of degree $|\mathbf{n}|$, which satisfy, by Euler's 
formula, the equation $x_{1} \partial_{1} g+\ldots+x_{d} \partial_{d} g=n g$. Taking derivative again shows easily that, if $g$ is a homogeneous polynomial of degree $n$, then

$$
\sum_{i=1}^{d} x_{i}^{2} \partial_{i}^{2} g+2 \sum_{1 \leq i<j \leq d} x_{i} x_{j} \partial_{i} \partial_{j} g=n(n-1) g,
$$

which applies, in particular, to $g \in H_{n}^{d}\left(W_{\gamma, 0}\right)$. On the other hand, by the item (i) of Lemma 2.10 and Lemma 2.11 $g \in H_{n}^{d}\left(W_{\gamma, 0}\right)$ satisfies the equation $L_{\gamma,-1} g=$ $-n(n+|\gamma|+d-1) g$, which is, by (1.2)

$$
\begin{aligned}
\sum_{i=1}^{d} x_{i}\left(1-x_{i}\right) \partial_{i}^{2} g & -2 \sum_{1 \leq i<j \leq d} x_{i} x_{j} \partial_{i} \partial_{j} g \\
& +\sum_{i=1}^{d}\left(\gamma_{i}+1-(|\gamma|+d) x_{i}\right) \partial_{i} g=-n(n+|\gamma|+d-1) g .
\end{aligned}
$$

Adding the two identities together and making use of the Euler's formula again, we conclude that $g \in H_{n}^{d}\left(W_{\gamma, 0}\right)$ satisfies the following equation

$$
\sum_{i=1}^{d} x_{i} \partial_{i}^{2} g+\sum_{i=1}^{d}\left(\gamma_{i}+1\right) \partial_{i} g=0
$$

Now, let $f(x)=(1-|x|) P_{\mathbf{n}}^{(\gamma, 1)}(x)$, where $P_{\mathbf{n}}^{(\gamma, 1)}(x) \in \mathcal{V}_{n-1}^{d}\left(W_{\gamma, 1}\right)$, and $g \in$ $H_{n}^{d}\left(W_{\gamma, 0}\right)$. Following the proof of Case 1 of the subsection 4.4 , we then arrive at (4.5). The summation over the derivatives of $g$, however, is exactly the differential operator in the left hand side of (4.16), so that $\langle f, g\rangle_{\gamma,-1}=0$.

\section{REFERENCES}

[1] M. Alfaro, F, Marcellán, M. L. Rezola, Estimates for Jacobi-Sobolev type orthogonal polynomials, Appl. Anal. 67 (1997), 157-174.

[2] M. Alfaro, M. Alvarez de Morales and M. L. Rezola, Orthogonality of the Jacobi polynomials with negative integer parameters, J. Comput. Appl. Math. 145 (2002), 379-386.

[3] M. Alfaro, T. E. Pérez, M. A. Piñar and M. L. Rezola, Sobolev orthogonal polynomials: The discrete-continuous case, Methods Appl. Anal. 6 (1999), 593-616.

[4] M. Alvarez de Morales, T.E. Perez and M.A. Piñar, Sobolev orthogonality for the Gegenbauer polynomials $\left\{C_{n}^{(-N+1 / 2)}\right\}_{n \geq 0}$, J. Comput. Appl. Math. 100 (1998) 111-120.

[5] K. Atkinson and O. Hansen, Solving the nonlinear Poisson equation on the unit disk, J. Integral Equations Appl.17 (2005), 223-241.

[6] C. F. Bracciali, A. M. Delgado, L. Fernández, T. E. Pérez and M. A. Piñar, New steps on Sobolev orthogonality in two variables, J. Comput. Appl. Math. 235 (2010), 916-926.

[7] C. F. Dunkl and Y. Xu, Orthogonal polynomials of several variables, Encyclopedia of Mathematics and its Applications, vol. 81, Cambridge University Press, 2001.

[8] L. Fernández, F. Marcellán, T. E. Pérez, M. A. Piñar, Recent trends on two variable orthogonal polynomials, in Differential algebra, complex analysis and orthogonal polynomials, 5986, Contemp. Math., 509, Amer. Math. Soc., Providence, RI, 2010.

[9] W. Gautschi, Orthogonal Polynomials: Computation and Approximation, Oxford Univ. Press, 2004.

[10] P. Iliev, Krall-Jacobi commutative algebras of partial differential operators, J. Math. Pures Appl. (9) 96 (2011), 446-461.

[11] K. H. Kwon and L. L. Littlejohn, Sobolev orthogonal polynomials and second-order differential equations, Rocky Mountain J. Math. 28 (1998), 547-594.

[12] J. K. Lee and L. L. Littlejohn, Sobolev orthogonal polynomials in two variables and second order partial differential equations, J. Math. Anal. Appl. 322 (2006), 1001-1017. 
[13] M. Piñar and Y. Xu, Orthogonal polynomials and partial differential equations on the unit ball, Proc. Amer. Math. Soc. 137 (2009), 2979-2987.

[14] T. Sauer, The Genuine Bernstein-Durrmeyer operator on a simplex, Results Math. 26 (1994), 99-130.

[15] G. Szegö, Orthogonal Polynomials, fourth ed., vol. 23, American Mathematical Society Colloquium Publications, Providence, 1975.

[16] S. Waldron, Multivariate Jacobi polynomials with singular weights, East J. Approx. 13 (2007), 163-183.

[17] Y. Xu, A family of Sobolev orthogonal polynomials on the unit ball, J. Approx. Theory 138 (2006), 232-241.

[18] Y. Xu, Sobolev orthogonal polynomials defined via gradient on the unit ball, J. Approx. Theory 152 (2008), 52-65.

Departments of Mathematics, Ankara University, Ankara 06100, Turkey

E-mail address: raktas@science.ankara.edu.tr

Department of Mathematics, University of Oregon, Eugene, Oregon 97403-1222.

E-mail address: yuan@math.uoregon.edu 